\title{
1 A fluorescent L-2-hydroxyglutarate biosensor
}

3 Zhaoqi Kang ${ }^{1}$, Manman Zhang ${ }^{3}$, Kaiyu Gao ${ }^{1}$, Wen Zhang ${ }^{4}$, Yidong Liu ${ }^{1}$, Dan Xiao ${ }^{1}$, Shiting

$4 \mathrm{Guo}^{1}$, Cuiqing $\mathrm{Ma}^{1}$, Chao $\mathrm{Gao}^{1, *}$, Ping $\mathrm{Xu}^{2, *}$

$6 \quad{ }^{1}$ State Key Laboratory of Microbial Technology, Shandong University, Qingdao, People's

$7 \quad$ Republic of China

${ }^{2}$ State Key Laboratory of Microbial Metabolism, Joint International Research Laboratory of

Metabolic \& Developmental Sciences, and School of Life Sciences \& Biotechnology,

Shanghai Jiao Tong University, Shanghai, People's Republic of China

${ }^{3}$ Tianjin Key Laboratory of Radiation Medicine and Molecular Nuclear Medicine,

Department of Radiobiology, Institute of Radiation Medicine of Chinese Academy of Medical

Science \& Peking Union Medical College, Tianjin, People's Republic of China

${ }^{4}$ Center for Gene and Immunotherapy, The Second Hospital of Shandong University, Jinan,

People's Republic of China

\section{*Corresponding authors:}

Mailing address for C. Gao: State Key Laboratory of Microbial Technology, Shandong

University, Qingdao 266237, People's Republic of China, Tel/Fax: +86-532-58631561, Email: jieerbu@sdu.edu.cn.

Mailing address for P. Xu: State Key Laboratory of Microbial Metabolism, and School of

Republic of China, Tel/Fax: +86-21-34206723, E-mail: pingxu@ sjtu.edu.cn. 


\section{Abstract}

L-2-Hydroxyglutarate (L-2-HG) plays important roles in diverse physiological processes, such as carbon starvation response, tumorigenesis, and hypoxic adaptation. Despite its importance and intensively studied metabolism, regulation of L-2-HG metabolism remains poorly understood and a regulator specifically responded to L-2-HG has never been identified. Based on the genomic neighborhood analysis of the gene encoding L-2-HG oxidase (LhgO), LhgR, which represses the transcription of $\operatorname{lhg} O$, was identified in Pseudomonas putida W619 in this study. LhgR was demonstrated to recognize L-2-HG as its specific effector molecule, and this allosteric transcription factor was then used as a biorecognition element for construction of L-2-HG-sensing FRET sensor. The newly developed L-2-HG sensor can conveniently monitor the concentrations of L-2-HG in various biological samples. In addition to bacterial L-2-HG generation during carbon starvation, biological functions of the L-2-HG dehydrogenase and hypoxia induced L-2-HG accumulation were also revealed by using the L-2-HG sensor in human cells. 
L-2-Hydroxyglutarate (L-2-HG) is an important metabolite in various domains of life. In mammals and plants, it is produced by lactate dehydrogenase (LDH) and malate dehydrogenase $(\mathrm{MDH})$-mediated 2-ketoglutarate $(2-\mathrm{KG})$ reduction under hypoxic conditions $^{1-5}$. In microorganisms, it is a metabolic intermediate of glutarate catabolism produced by a glutarate hydroxylase, $\mathrm{CsiD}^{6-8}$. L-2-HG dehydrogenase (L2HGDH) or L-2-HG oxidase (LhgO), an FAD-containing oxidoreductase that converts L-2-HG to 2-KG, plays an indispensable role in the catabolism of $\mathrm{L}-2-\mathrm{HG}^{9}$. While extensive efforts have been devoted to investigate L-2-HG anabolism and catabolism, the molecular machinery that specifically senses L-2-HG and regulates its metabolism has not been clarified until now.

L-2-HG is an inhibitor of 2-KG dependent dioxygenases with specific pro-oncogenic capabilities ${ }^{10,11}$. Thus, this oncometabolite is viewed as a biomarker for a variety of cancers and its rapid and sensitive measurement in body fluids is of clinical significance ${ }^{12-15}$. Importantly, L-2-HG also has endogenous functions in healthy animal cells. For example, this compound was recently identified to aid in the proliferation and antitumorigenic abilities of coordinate glycolytic flux with epigenetic modifications ${ }^{17}$. Considering the diversity of the roles of L-2-HG in cell metabolism, development and optimization of assays for real-time tracking of this metabolite in living cells is required. chromatography-tandem mass spectrometry (GC-MS/MS) $)^{20,21}$ are often used to assess the extracellular concentrations of L-2-HG. These common methods are, at present, time 
consuming, expensive to perform, and require highly skilled personnel. In addition, these

61 destructive methods are also incompatible with real-time monitoring of the fluctuations of L2-HG concentrations in intact living cells. In this study, we identified and characterized LhgR, an L-2-HG catabolism regulator in Pseudomonas putida W619. Mechanistically, LhgR represses the transcription of $\mathrm{LhgO}$ encoding gene $\operatorname{lhg} \mathrm{O}$. L-2-HG is a specific effector molecule of LhgR and prevents LhgR binding to the promoter region of $\operatorname{lhg} O$. Then, we report the development and application of the LhgR based L-2-HG biosensor via Förster resonance energy transfer (FRET), a technology widely applied in the investigation of temporal dynamics of various small molecules, such as potassium ${ }^{22,23}$, glycine ${ }^{24}$, and cAMP $^{25,26}$. The newly developed sensor was used to test L-2-HG concentrations in various biological samples and achieved competitive accuracy and precision. We also took advantage of this biosensor to test predictions about the carbon starvation-induced L-2-HG production in bacteria and to demonstrate hypoxia-induced L-2-HG production by LDH and MDH in human cells. Therefore, the biosensor can also act as a useful tool for real-time measurement of the L-2-HG concentrations in living cells.

\section{Results}

\section{LhgR regulates L-2-HG catabolism}

In this study, bacteria containing LhgO encoding gene $\operatorname{lhg} O$ were selected to study the regulation of L-2-HG metabolism. Homologs of LhgO can be found in 612 diverse bacterial strains. Similarly organized chromosomal clusters can be found in many bacterial genomes, which contain various combinations of genes related to glutarate metabolism (csiD, $\operatorname{lhg} O$, 
gabT, gabD, and gabP) (Fig. 1a). In Pseudomonas putida KT2440, the glutarate regulon is regulated by allosteric transcription factor CsiR, which is encoded upstream of $c s i D^{27}$. The glutarate sensing allosteric transcription factor CsiR and its cognate promoter have been cloned into broad host range vectors to create a glutarate biosensor ${ }^{28}$. Interestingly, a different pattern of $\operatorname{lhg} O$ gene neighborhood is observed in a few species that do not contain $c s i D$ homologs (Fig. 1a). For example, a gene encoding a GntR family protein, $\operatorname{lhg} R$, is found directly upstream of $l h g O$ in $P$. putida $\mathrm{W} 619$. The absence of $c s i D$ gene related to glutarate catabolism made us to anticipate that $l h g O$ of $P$. putida W619 might be solely involved in L2-HG metabolism and be L-2-HG inducible.

The $\operatorname{lhg} O$ gene in $P$. putida W619 was cloned into pME6032 vector, and the resulting plasmid was transferred into P. putida KT2440 ( $\Delta l h g O)$. As shown in Fig. 1b-c, complement of $\operatorname{lhg} O$ in $P$. putida W619 could restore glutarate and L-2-HG utilization abilities of $P$. putida $\mathrm{KT} 2440(\Delta \operatorname{lhg} O)$, confirming that $\operatorname{lhg} O$ encodes a functional L-2-HG catabolic enzyme. To identify the function of LhgR in P. putida W619, the gene segment F2-lhgR-F1$\operatorname{lng} O$, which contains the promoter of $\operatorname{lng} R(\mathrm{~F} 2), \operatorname{lng} R$, the promoter of $\operatorname{lng} O(\mathrm{~F} 1)$, and $\operatorname{lhg} O$, was cloned into pME6032 vector, and the resulting plasmid was transferred into different derivatives of $P$. putida KT2440 (Fig. 1d). As shown in Fig. 1e, exogenous L-2-HG, but not its mirror-image enantiomer D-2-HG, can induce the expression of $\operatorname{lhg} O$ in the gene segment F2-lhgR-F1-lhgO and restore $\mathrm{LhgO}$ activity in $P$. putida KT2440 ( $\Delta c s i R \Delta l h g O)$. In addition, the activity of $\mathrm{LhgO}$ was also detected in $P$. putida KT2440 ( $\Delta c s i R \Delta l h g O)$ harboring pME6032-F2-lhgR-F1-lhgO when cultured with glutarate as the sole carbon source. However, no activity of $\mathrm{LhgO}$ was detected in P. putida KT2440 ( $\Delta c s i R \Delta c s i D \Delta l h g O)$, in 
which the key gene responsible for L-2-HG production from glutarate was deleted. These results indicated that LhgR represses the expression of LhgO and L-2-HG, but nor D-2-HG or glutarate, might be the effector molecule of LhgR.

\section{LhgR specifically responds to L-2-HG}

To determine whether LhgR directly interacts with the promoter region of $l h g O$, LhgR in $P$. putida W619 was overexpressed in E. coli BL21(DE3) and purified by Ni-chelating chromatography (Fig. 2a). Based on the results of gel filtration and sodium dodecyl sulfatepolyacrylamide gel electrophoresis (SDS-PAGE), LhgR behaved as a dimer (Fig. 2b). Subsequently, electrophoretic mobility shift assays (EMSAs) were conducted using $\operatorname{lh} g O$ promoter $(\mathrm{F} 1)$ and purified LhgR. As shown in Fig. 2c, LhgR bound to F1 in a concentrationdependent manner. LhgR completely shifted fragment F1 when an 8-fold molar excess was used. A DNase I footprinting assay was also performed using purified LhgR and fragment F1. A protected region containing palindromic $\mathrm{N}_{\mathrm{y}} \mathbf{G T N} \mathrm{T}_{\mathrm{x}} \mathbf{A C N} \mathrm{N}_{\mathrm{y}}$ consensus binding motif of GntR-

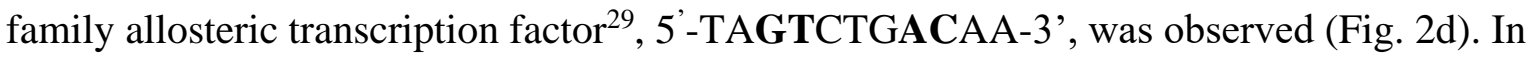
addition, LhgR also bound to its promoter (F2) in EMSAs and a similar consensus binding motif, 5'-TTGTCTGACAA-3', was protected in DNase I footprinting assay (Supplementary Fig. 1a-b).

$$
\text { The effects of L-2-HG, D-2-HG, glutarate, 2-KG, L-lysine, 5-aminovalerate, and }
$$
succinate on $\operatorname{LhgR}$ binding to the $\operatorname{lhg} O$ promoter region F1 were also assessed by EMSAs. The release of LhgR from fragments F1 was observed only in the presence of L-2-HG (Fig. 2e). These results indicated that L-2-HG can specifically prevent the binding of LhgR to the 
promoter of $\operatorname{lhg} O$ and induce its expression. In particular, LhgR might self-repress its expression and L-2-HG can also contribute in inducing the expression of $l h g R$ (Supplementary Fig. 1c).

\section{Design and optimization of the L-2-HG-sensing reporter}

FRET sensors, which combine a ligand-binding moiety and a pair of donor-acceptor fluorescent pair, allow measurement of ligand concentrations based on the ligand-binding induced changes of FRET efficiency ${ }^{22-26}$. In this study, the L-2-HG-sensing fluorescent reporter (LHGFR) was constructed by fusion of the optimized cyan and yellow fluorescent protein variants, mTFP $^{30}$ and Venus ${ }^{31}$, to the $\mathrm{N}$ - and C-terminus of LhgR (Supplementary Fig. 2). This first LHGFR was named as LHGFR ${ }_{0 N 0 C}$, where the subscript indicates the number of amino acids truncated from the N- and C-terminus of LhgR. Subsequently, LHGFR ${ }_{0 N 0 C}$ was overexpressed in E. coli BL21(DE3) and purified by a Ni-chelating chromatographic column (Supplementary Fig. 3). The addition of L-2-HG could reduce the emission peak at $492 \mathrm{~nm}$ of mTFP and increase the emission peak at $526 \mathrm{~nm}$ of Venus (Supplementary Fig. 4), indicating that L-2-HG binding to LHGFRoNoC increases FRET between the fluorophores (Fig. 3a). In addition, L-2-HG increased the emission ratio of Venus to mTFP in a dose-dependent manner, with a maximum ratio change $\left(\Delta R_{\max }\right)$ of $11.47 \pm$ $0.38 \%$, an apparent dissociation constant $\left(K_{d}\right)$ of $2.74 \pm 0.73 \mu \mathrm{M}$, and a Hill slope close to 1 (Fig. 3b).

To increase the magnitude of responses, LHGFR was optimized by truncating N- and Cterminal amino acids of LhgR or by adding a series of artificial linkers between LhgR and 
various fluorescent proteins ${ }^{23,32-34}$ (Fig. 3c and Supplementary Fig. 5). Truncation of just three to seven C-terminal amino acids in LhgR could significantly increase $\Delta R_{\max }$ of the sensor (Fig. 3c-d and Supplementary Fig. 5). Among the five sensors with increased response magnitude values, LHGFRon7c was the best mutant with a higher $\Delta R_{\max }$ of $60.37 \pm 1.30 \%$ (Fig. 3d and Supplementary Fig. 6a-e). In addition, LHGFRon3C was also a promising sensor with a high $\Delta R_{\max }$ of $56.13 \pm 0.29 \%$ and a high $K_{d}$ of $29.33 \pm 1.24 \mu \mathrm{M}$ (Supplementary Fig. 6a). The unique $K_{d}$ of LHGFRoN3C might make this sensor usable in monitoring L-2-HG at a high dynamic range.

Then, a set of intermediates of TCA cycle and L-lysine catabolism were used to examine the specificity of LHGFRoN3C and LHGFRon7c. None of oxaloacetate, citrate, isocitrate, 2KG, succinate, fumarate, cis-aconitate, L-malate, pyruvate, L-lysine, 5-aminovalerate, glutarate, D-lactate, and L-lactate induced emission ratio changes in LHGFRoN3C or LHGFRoN7C (Fig. 3e-f). Although D-2-HG elicited small changes in the emission ratio of LHGFRoN7C at a concentration of $240 \mu \mathrm{M}$, dose-response curves of LHGFRon3C and LHGFRoN7C for D-2-HG revealed insignificant binding of D-2-HG and $K_{d}$ values much higher than that for L-2-HG (Supplementary Fig. 7a-b). L-2-HG-dependent emission ratio changes of LHGFRoN3C or LHGFRoN7C were significantly reversible by L-2-HG oxidation catalyzed by 5 $\mu \mathrm{M}$ LhgO (Fig. 3g-h) and both biosensors were stable for the detection of L-2-HG from $\mathrm{pH}$ 6.0 to 8.0 (Supplementary Fig. 8a-b). The high specificity, reversibility, and stability of LHGFRoN3C and LHGFRoN7C indicated the availability of these biosensors in monitoring dynamic changes in L-2-HG under physiological conditions. 


\section{Characterization of LHGFR in biological samples}

Next, we investigated whether LHGFR could be used to quantify L-2-HG concentrations in different biological samples. When L-2-HG with increasing concentrations (0 to $2 \mathrm{mM}$ ) were added into serum and urine samples of healthy adults, the response curves were nearly identical with that in assay buffer for both LHGFR ${ }_{0 N 3 C}$ and LHGFR 0 N7C (Fig. 4a-d, Supplementary Fig. 6a and Fig. 6e). Thus, quantitative determination of L-2-HG could be conducted by mixing the target samples with LHGFR and measuring the emission ratios with a conventional fluorescence microplate reader. Based on the response curves established for L-2-HG quantification, both biosensors were used to assay the concentrations of L-2-HG in human serum and urine (Fig. 4e-f). The results of LHGFR0N3C and LHGFR0N7C showed close agreement with the results of LC-MS/MS, the current standard method for clinical assays of L-2-HG. Besides its accuracy and precision in L-2-HG quantification (Supplementary Table 1), LHGFR0N7C also exhibited superior applicability in its lower limits of detection (LOD) (Supplementary Table 2).

In a previous report, L-2-HG was confirmed to be a metabolic intermediate of glutarate metabolism in P. putida KT24407 ${ }^{7}$ LHGFR0N3C and LHGFR0N7C also exhibited high accuracy and precision in quantification of L-2-HG in bacterial culture medium (Fig. 4g-i). When cultured in medium containing $20 \mathrm{mM}$ glucose and $5 \mathrm{mM}$ glutarate as carbon sources, the growth of $P$. putida KT2440 ( $\Delta l h g O)$ was significantly delayed which might be due to the possible toxicity of accumulated L-2-HG (Fig. 4j). Nearly identical results of L-2-HG quantification were also obtained by either using LHGFR0N3C or LHGFR0N7C (Fig. 4k-1). Mutual corroboration between the two biosensors further confirmed their applicabilities in in 
vitro $\mathrm{L}-2-\mathrm{HG}$ quantification of various biological samples.

\section{Monitoring L-2-HG fluctuations in living bacteria by LHGFR}

We investigated whether the FRET-based L-2-HG sensor LHGFR could detect possible variations of L-2-HG in living bacteria. The biosensor LHGFR $_{0 \mathrm{~N} 3 \mathrm{C}}$ was expressed in E. coli BL21(DE3). Exogenous L-2-HG was added to the culture system of E. coli BL21(DE3) to achieve concentrations between 0 and $20 \mathrm{mM}$ and the emission ratio was continuously recorded. As shown in Fig. 5a, exogenous L-2-HG at concentrations lower than $10 \mathrm{mM}$ could increase emission ratio in a dose-dependent manner. The apparent $K_{d}$ of LHGFR LN3C expressed in E. coli BL21(DE3) was determined to be $888.47 \pm 24.74 \mu \mathrm{M}$ by fitting emission ratios against exogenous L-2-HG concentrations (Fig. 5b). Thus, the intracellular L-2-HG concentrations might be approximately 30 -fold lower than the external supply, assuming that the affinity of LHGFR is not affected in the cytosolic environment ${ }^{35}$. The specificity of LHGFR $_{0 \text { N3C }}$ expressed in E. coli BL21(DE3) was also characterized. As shown in Fig. 5c, only exogenous L-2-HG could significantly increase the emission ratio in E. coli BL21(DE3), while glutarate, D-2-HG, and glucose could not.

Besides being a metabolic intermediate of exogenous glutarate catabolism in P. putida KT2440, L-2-HG is also reported as a metabolite produced from endogenous L-lysine during carbon starvation of $E$. coli ${ }^{36,37}$. Thus, whether carbon starvation could induce intracellular L2-HG accumulation of E. coli was investigated. As shown in Fig. 5d, no change in the emission ratio was detected when $20 \mathrm{mM}$ glucose was added to the culture system. However, the emission ratio increased during carbon starvation of E. coli BL21(DE3), suggesting that 
carbon starvation induced L-2-HG production. The emission ratios also increased after culturing E. coli cells for $6 \mathrm{~h}$ with glucose addition (Fig. 5e), which might be due to carbon starvation induced by depletion of exogenous glucose. In addition, the emission ratio increased at the beginning of carbon starvation, reaching a maximum value at $3 \mathrm{~h}$ and then decreased to initial levels at $8 \mathrm{~h}$ (Fig. 5e). These results confirmed that L-2-HG is a temporary metabolite during carbon starvation and LHGFRoN3C can indeed detect a change in intracellular L-2-HG concentrations in real time.

To identify whether carbon starvation induced endogenous L-2-HG production also results from the glutarate hydroxylase activity of CsiD, gene $c s i D$ was disrupted and LHGFRon3C was expressed in E. coli MG1655(DE3). Gene lhgO in E. coli MG1655(DE3) was also disrupted to investigate its role in endogenous L-2-HG catabolism. As expected, the emission ratio of LHGFR $0 \mathrm{~N}_{3 \mathrm{C}}$ in $E$. coli MG1655(DE3) $(\Delta c s i D)$ remained unaffected during carbon starvation, whereas disruption of $\operatorname{lhg} O$ significantly increased the emission ratio of LHGFRon3C in E. coli MG1655(DE3) ( $\Delta l h g O)$ (Fig. 5f), confirming the roles of CsiD and LhgO in endogenous L-2-HG catabolism during carbon starvation. The performance of LHGFRoN7C in monitoring L-2-HG fluctuations in living bacteria was also studied and similar results were acquired (Supplementary Fig. 9).

\section{Monitoring L-2-HG production in human cells by LHGFR}

Next, both the biosensors were expressed in HEK293FT cells to monitor L-2-HG levels inside living human cells. As shown in Fig. 6a and Supplementary Fig. 10a, both biosensors were successfully expressed in HEK293FT cells, mainly localized in the cytosol. LHGFRoN3C 
and LHGFRon7c were calibrated by adding increasing concentrations ( 0 to $10 \mathrm{mM})$ of L-2HG to $10 \mu \mathrm{M}$ digitonin-permeabilized HEK293FT cells. The apparent $K_{d}$ of LHGFRoN3C and LHGFRon7c expressed in HEK293FT cells were $43.79 \pm 3.05 \mu \mathrm{M}$ and $9.35 \pm 0.67 \mu \mathrm{M}$, respectively (Fig. 6b and Supplementary Fig. 10b). Based on the emission ratio of nonpermeabilized HEK293FT cells under physiological conditions, the basal L-2-HG concentration was determined to be $22.95 \pm 11.22 \mu \mathrm{M}$ in LHGFRon3c-expressing cells (Fig. 6b), and $20.24 \pm 8.39 \mu \mathrm{M}$ in LHGFRon7c-expressing cells (Supplementary Fig. 10b). As shown in Fig. 6c and Supplementary Fig. 10c, only exogenous L-2-HG could significantly increase the emission ratio in $10 \mu \mathrm{M}$ digitonin-permeabilized HEK293FT, further suggesting the high specificity of these two biosensors.

LHGFRoN3C and LHGFRon7c were then used to detect L-2-HG fluctuations associated with the loss-of-function of L2HGDH, the only reported enzyme which can catabolize L-2HG in human cells. HEK293FT cells were co-transfected with siRNA targeting L2HGDH and LHGFRon3C or LHGFRon7c. As shown in Fig. 6d and Supplementary Fig. 10d, the transfection of siRNA targeting L2HGDH increased the emission ratio of both the biosensors, indicating an accumulation of intracellular L-2-HG due to a decrease in L2HGDH levels (Fig. 6d and Supplementary Fig. 10d).

The sensitivity of LHGFR to changes in hypoxia-induced production of L-2-HG was also studied. LHGFRon3C, which has a wide detection range due to its high $K d$, was used for this purpose. The emission ratio of LHGFRon3C in HEK293FT cells after $24 \mathrm{~h}$ exposure to $2 \%$ oxygen was higher than the ratio obtained under normoxic conditions and hypoxia induced a 3.5-fold increase of L-2-HG (Fig. 6e). In addition, exogenous cell-permeable 
dimethyl-2-ketoglutarate significantly increased the emission ratio of LHGFRoN3C under hypoxic conditions, suggesting that hypoxia-induced L-2-HG might originate from 2-KG (Fig. 6e).

LDHA and MDH2 have been reported to participate in hypoxia-induced L-2-HG production due to their "promiscuous" catalytic activities ${ }^{1}$. In support of this conclusion, siRNAs targeting LDHA and MDH2 were transfected separately or in combination into LHGFRon3c-expressing HEK293FT cells. As shown in Fig. 6f, decrease of LDHA and MDH2 significantly reduced the emission ratio of LHGFRoN3C under hypoxic conditions, suggesting that these enzymes indeed contribute to the production of L-2-HG from 2-KG.

\section{Discussion}

Bacteria have evolved to respond and catabolize a wide range of metabolites. The availability of genomic information from different organisms provided researchers with a new route to develop robust predictions for new transcriptional regulators and their physiological functions. Here, we used a genomic neighborhood analysis approach combined with genetic and biochemical techniques to uncover a novel transcriptional regulator of L-2-HG catabolism. The identified transcriptional regulator, LhgR, was present directly upstream of LhgO in P. putida W619 (Fig. 1a). It specifically binds as a dimer to the promoter region of $\operatorname{lhg} O$ and represses the transcription of $\operatorname{lhg} O$ gene. L-2-HG interferes with the DNA-binding activity of $\mathrm{LhgR}$ and induces expression of $\mathrm{LhgO}$. To our knowledge, $\mathrm{LhgR}$ is the first known example of an allosteric transcription factor which specially responses to L-2-HG in all domains of life. This finding showed the application of a collection of sequenced genomes 
in identification of novel transcriptional regulators and the approach can be expanded to target other transcriptional regulators in diverse bacteria.

L-2-HG is a harbinger of altered metabolism and participates in the pathogenesis of L-2hydroxyglutaric acidurias and cancer ${ }^{12-15,38}$. Standard methods to measure L-2-HG are based on MS-based techniques which are time consuming and requires expensive highly skilled workers. LhgR can bind to L-2-HG and then undergo conformational changes, which in turn affects DNA-binding. Thus, a FRET biosensor, LHGFRonoc, utilizing the allosteric transcription factor LhgR as an L-2-HG biorecognition element was constructed for a convenient assay of L-2-HG concentrations. As a ratiometric sensor, the emission ratio changes of LHGFR is not affected by the amount of sensor in biological samples or in living cells, and thus allows more accurate measurements. The biosensor was optimized by truncating the $\mathrm{N}$ - and C-terminal domains of LhgR or by adding an artificial linker to the $\mathrm{N}$ and C-terminal regions of LhgR. The optimized variants, LHGFRon3C and LHGFRon7c, increased $\Delta R_{\max }$ from $11.47 \pm 0.38 \%$ to $56.13 \pm 0.29 \%$ and $60.37 \pm 1.30 \%$, respectively, significantly improving the sensitivity for L-2-HG detection (Fig. 3b, Supplementary Fig. 6a and Fig. 6e). Besides signal recognition, signal transduction is also an essential aspect for the development of biosensors. Various biosensing systems, including CRISPR-Cas12a- and allosteric transcription factors-mediated small molecule detector ${ }^{39}$, allosteric transcription factors-based nicked DNA-template-assisted signal transduction ${ }^{40}$, and quantum-dotallosteric transcription factors-FRET ${ }^{41}$, have been reported. Other biosensors based on L-2HG responding to LhgR and the corresponding transduction mechanisms could also be developed for detection of L-2-HG. 
LHGFR $_{0 \mathrm{~N} 3 \mathrm{C}}$ and LHGFR 0 7C to varying concentrations of L-2-HG in artificial urine and

serum (prepared through addition of L-2-HG to the serum and urine of healthy adults) were

patients with L-2-hydroxyglutaric aciduria and L-2-HG-associated brain malignancies is about

LHGFR $_{0 \mathrm{~N} 7 \mathrm{C}}$, requires simply mixing traces of serum with LHGFR and then detecting changes in ratios using a fluorescence microplate reader. Besides higher detection sensitivity, LHGFR also has superior accuracy and precision over previous MS-based methods for L-2HG detection (Fig. 4 and Supplementary Table 1). Being genetically-encoded, L-2-HG sensors can be produced in great quantities by recombinant bacteria with low cost and could be applied in future rapid and sensitive clinical diagnosis of L-2-HG-related diseases. curves construction. The calibrated in vivo response curves of both biosensors in digitoninpermeabilized HEK293FT cells gave similar $K_{d}$ of those in in vitro assays. Based on the in vivo response curves of LHGFR0N3C or LHGFR0N7C, the basal L-2-HG concentration in HEK293FT cells under physiological conditions was $22.95 \pm 11.22 \mu \mathrm{M}$ or $20.24 \pm 8.39 \mu \mathrm{M}$, respectively (Fig. 6b and Supplementary Fig. 10b). L-2-HG quantification in living 
approaches after complicated sample handling and data analysis ${ }^{2,3}$. Considering the fact that hypoxic cells, activated T-cells, and certain cancer cells might accumulate L-2-HG to high concentrations $^{2-4,12-14,16,43}$, LHGFRoN3C with high $K_{d}$ and detection range might be a more viable alternative for the in vivo assay of L-2-HG concentrations.

The potential of LHGFR for real-time monitoring of fluctuations in intracellular L-2-HG concentrations was illustrated by using bacterial cells and HEK293FT cells. It was revealed that carbon starvation also induced temporary intracellular accumulation of L-2-HG in E. coli cells. CsiD and $\mathrm{LhgO}$ played indispensable roles in endogenous anabolism and catabolism of L-2-HG, respectively (Fig. $5 f$ and Supplementary Fig. 9f). In addition, it was identified that growth of the strain containing $\operatorname{lhg} O$ mutation was inhibited when high levels of L-2-HG was present (Fig. 4j-1). Besides being a pathogenic metabolite inducing various cancers and L-2hydroxyglutaric aciduria in humans ${ }^{12-15,38,42}$, L-2-HG would also be a toxic metabolite to bacterial cells. L-2-HG catabolizing enzymes, including L2HGDH in humans ${ }^{9}$, dL2HGDH in Drosophila ${ }^{17}$, and LhgO in P. putida ${ }^{7,8}$ and E. coli ${ }^{6,44}$, might all exist as detoxification proteins of L-2-HG. The functions of L2HGDH in L-2-HG catabolism and LDHA and MDH2-mediated 2-KG reduction in hypoxia-induced L-2-HG production were also confirmed in HEK293FT cells using LHGFR as an indicator of L-2-HG. LHGFR can be added in the emerging list of metabolite sensors that have been established in mammalian cells, such as probes for ATP ${ }^{45}$, acetylcholine ${ }^{46}$, glycine ${ }^{24}$, and $\mathrm{NAD}^{+} / \mathrm{NADH}^{47}$. Several genetically encoded fluorescent metabolite sensors, like the NAD $/$ NADH probe SoNar, have been successfully applied in the screening of anti-tumor agents ${ }^{47}$. L-2-HG has been exploited as a potential therapeutic target in renal cancer ${ }^{14}$ or a biomarker for cancer diagnosis and 
prognostic assessment ${ }^{15,48}$. The L-2-HG biosensors might also be utilized in the diagnosis and screening of anti-tumor agents for L-2-HG-related cancer.

In summary, a regulatory protein $\mathrm{LhgR}$, which is involved in L-2-HG catabolism and specifically responds to L-2-HG, was identified in P. putida W619. Then, two FRET-based L2-HG biosensors, LHGFRoN3C and LHGFRoN7c, with excellent sensitivity, specificity, and stability, were constructed. The methods for quantitative estimation of L-2-HG concentrations in various biological samples and living cells by using L-2-HG biosensors were also established. We expect these L-2-HG biosensors to be of practical interest in future research on metabolism of L-2-HG and the diagnosis and treatment of L-2-HG-related diseases.

\section{Methods}

Methods, including statements of data availability and any associated accession codes and references, are available in the online version of the paper.

\section{References}

1. Linster, C.L., Van Schaftingen, E. \& Hanson, A.D. Metabolite damage and its repair or pre-emption. Nat. Chem. Biol. 9, 72-80 (2013).

2. Intlekofer, A.M. et al. Hypoxia induces production of L-2-hydroxyglutarate. Cell Metab. 22, 304-311 (2015).

3. Oldham, W.M., Clish, C.B., Yang, Y. \& Loscalzo, J. Hypoxia-mediated increases in L-2hydroxyglutarate coordinate the metabolic response to reductive stress. Cell Metab. 22, 291-303 (2015). 
4. Harris, A.L. A new hydroxy metabolite of 2-oxoglutarate regulates metabolism in hypoxia. Cell Metab. 22, 198-200 (2015).

5. Hüdig, M. et al. Plants possess a cyclic mitochondrial metabolic pathway similar to the mammalian metabolic repair mechanism involving malate dehydrogenase and L-2hydroxyglutarate dehydrogenase. Plant Cell Physiol. 56, 1820-1830 (2015).

6. Knorr, S. et al. Widespread bacterial lysine degradation proceeding via glutarate and L-2hydroxyglutarate. Nat. Commun. 9, 5071 (2018).

7. Zhang, M. et al. Increased glutarate production by blocking the glutaryl-CoA dehydrogenation pathway and a catabolic pathway involving L-2-hydroxyglutarate. Nat. Commun. 9, 2114 (2018).

8. Thompson, M.G. et al. Massively parallel fitness profiling reveals multiple novel enzymes in Pseudomonas putida lysine metabolism. mBio 10, e02577-18 (2019).

9. Rzem, R. et al. A gene encoding a putative FAD-dependent L-2-hydroxyglutarate dehydrogenase is mutated in L-2-hydroxyglutaric aciduria. Proc. Natl. Acad. Sci. USA 101, 16849-16854 (2004).

10. Xu, W. et al. Oncometabolite 2-hydroxyglutarate is a competitive inhibitor of $\alpha$ ketoglutarate-dependent dioxygenases. Cancer Cell 19, 17-30 (2011).

11. Chowdhury, R. et al. The oncometabolite 2-hydroxyglutarate inhibits histone lysine demethylases. EMBO Rep. 12, 463-469 (2011).

12. Shim, E.H. et al. L-2-Hydroxyglutarate: an epigenetic modifier and putative oncometabolite in renal cancer. Cancer Discov. 4, 1290-1298 (2014).

13. Ye, D., Guan, K.L. \& Xiong, Y. Metabolism, activity, and targeting of D- and L-2- 
hydroxyglutarates. Trends Cancer 4, 151-165 (2018).

14. Shelar, S. et al. Biochemical and epigenetic insights into L-2-hydroxyglutarate, a potential therapeutic target in renal cancer. Clin. Cancer Res. 24, 6433-6446 (2018).

15. Wang, J.H. et al. Prognostic significance of 2-hydroxyglutarate levels in acute myeloid leukemia in China. Proc. Natl. Acad. Sci. USA 110, 17017-17022 (2013).

16. Tyrakis, P.A. et al. S-2-hydroxyglutarate regulates $\mathrm{CD} 8^{+}$T-lymphocyte fate. Nature 540, 236-241 (2016)

17. Li, H. et al. Drosophila larvae synthesize the putative oncometabolite L-2hydroxyglutarate during normal developmental growth. Proc. Natl. Acad. Sci. USA 114, $1353-1358$ (2017).

18. Struys, E.A., Jansen, E.E., Verhoeven, N.M. \& Jakobs, C. Measurement of urinary Dand L-2-hydroxyglutarate enantiomers by stable-isotopedilution liquid chromatography-tandem mass spectrometry after derivatization with diacetyl-L-tartaric anhydride. Clin. Chem. 50, 1391-1395 (2004).

19. Calderón, C., Horak, J. \& Lämmerhofer, M. Chiral separation of 2-hydroxyglutaric acid on cinchonan carbamate based weak chiral anion exchangers by high-performance liquid chromatography. J. Chromatogr. A 1467, 239-245 (2016).

20. Fernández-Galán, E. et al. Validation of a routine gas chromatography mass spectrometry method for 2-hydroxyglutarate quantification in human serum as a screening tool for detection of idh mutations. J. Chromatogr. B 1083, 28-34 (2018).

21. Strain, S.K., Groves, M.D., Olino, K.L. \& Emmett, M.R. Measurement of 2hydroxyglutarate enantiomers in serum by chiral gas chromatography-tandem mass 

spectrometry and its application as a biomarker for IDH mutant gliomas. Clin. Mass Spectrom. 15, 16-24 (2020).

22. Wang, S. et al. Potassium channel selectivity filter dynamics revealed by single-molecule FRET. Nat. Chem. Biol. 15, 377-383 (2019).

23. Bischof, H. et al. Novel genetically encoded fluorescent probes enable real-time detection of potassium in vitro and in vivo. Nat. Commun. 8, 1422 (2017).

24. Zhang, W.H. et al. Monitoring hippocampal glycine with the computationally designed optical sensor GlyFS. Nat. Chem. Biol. 14, 861-869 (2018).

25. Surdo, N.C. et al. FRET biosensor uncovers cAMP nano-domains at $\beta$-adrenergic targets that dictate precise tuning of cardiac contractility. Nat. Commun. 8, 15031 (2017).

26. Mukherjee, S. et al. A novel biosensor to study cAMP dynamics in cilia and flagella. eLife 5, e14052 (2016).

27. Zhang, M. et al. Regulation of glutarate catabolism by GntR family regulator CsiR and LysR family regulator GcdR in Pseudomonas putida KT2440. mBio 10, e01570-19 (2019).

28. Thompson, M.G. et al. Robust characterization of two distinct glutarate sensing transcription factors of Pseudomonas putida L-lysine metabolism. ACS Synth. Biol. 8, 2385-2396 (2019).

29. Rigali, S., Derouaux, A., Giannotta, F. \& Dusart, J. Subdivision of the helix-turn-helix GntR family of bacterial regulators in the FadR, HutC, MocR, and YtrA subfamilies. $J$. Biol. Chem. 277, 12507-12515 (2002).

30. Day, R.N., Booker, C.F. \& Periasamy, A. Characterization of an improved donor 

031203 (2008).

31. Nagai, T. et al. A variant of yellow fluorescent protein with fast and efficient maturation for cell-biological applications. Nat. Biotechnol. 20, 87-90 (2002).

32. Hires, S.A., Zhu, Y. \& Tsien, R.Y. Optical measurement of synaptic glutamate spillover and reuptake by linker optimized glutamate-sensitive fluorescent reporters. Proc. Natl. Acad. Sci. USA 105, 4411-4416 (2008).

33. Chen, X., Zaro, J.L. \& Shen, W.C. Fusion protein linkers: property, design and functionality. Adv. Drug Deliv. Rev. 65, 1357-1369 (2013).

34. Steffen, V. et al. A toolbox of genetically encoded FRET-based biosensors for rapid Llysine analysis. Sensors 16, 1604 (2016).

35. Kaper, T., Lager, I., Looger, L.L., Chermak, D. \& Frommer, W.B. Fluorescence resonance energy transfer sensors for quantitative monitoring of pentose and disaccharide accumulation in bacteria. Biotechnol. Biofuels 1, 11 (2008).

36. Marschall, C. et al. Molecular analysis of the regulation of $c s i D$, a carbon starvationinducible gene in Escherichia coli that is exclusively dependent on $\sigma^{\mathrm{S}}$ and requires activation by cAMP-CRP. J. Mol. Biol. 276, 339-353 (1998).

37. Metzner, M., Germer, J. \& Hengge, R. Multiple stress signal integration in the regulation of the complex $\sigma^{\mathrm{S}}$-dependent $c$ siD-ygaF-gabDTP operon in Escherichia coli. Mol. Microbiol. 51, 799-811 (2004).

38. Kranendijk, M., Struys, E.A., Salomons, G.S., Van der Knaap, M.S. \& Jakobs, C. Progress in understanding 2-hydroxyglutaric acidurias. J. Inherit. Metab. Dis. 35, 
$571-587(2012)$

39. Liang, M. et al. A CRISPR-Cas12a-derived biosensing platform for the highly sensitive detection of diverse small molecules. Nat. Commun. 10, 3672 (2019).

40. Cao, J. et al. Harnessing a previously unidentified capability of bacterial allosteric transcription factors for sensing diverse small molecules in vitro. Sci. Adv. 4, eaau4602 (2018).

41. Grazon, C. et al. A progesterone biosensor derived from microbial screening. Nat. Commun. 11, 1276 (2020).

42. Moroni, I. et al. L-2-Hydroxyglutaric aciduria and brain malignant tumors: a predisposing condition? Neurology 62, 1882-1884 (2004).

43. Rakheja, D. et al. Papillary thyroid carcinoma shows elevated levels of 2hydroxyglutarate. Tumour Biol. 32, 325-333 (2011).

44. Kalliri, E., Mulrooney, S.B. \& Hausinger, R.P. Identification of Escherichia coli YgaF as an L-2-hydroxyglutarate oxidase. J. Bacteriol. 190, 3793-3798 (2008).

45. Lobas, M.A. et al. A genetically encoded single-wavelength sensor for imaging cytosolic and cell surface ATP. Nat. Commun. 10, 711 (2019).

46. Jing, M. et al. A genetically encoded fluorescent acetylcholine indicator for in vitro and in vivo studies. Nat. Biotechnol. 36, 726-737 (2018).

47. Zhao, Y. et al. SoNar, a highly responsive $\mathrm{NAD}^{+} / \mathrm{NADH}$ sensor, allows high-throughput metabolic screening of anti-tumor agents. Cell Metab. 21, 777-789 (2015).

48. Yong, C., Stewart, G.D. \& Frezza, C. Oncometabolites in renal cancer. Nat. Rev. Nephrol. 16, 156-172 (2019). 
Acknowledgements

This work was supported by the grants of National Key R\&D Program of China (2019YFA0904800, 2018YFA0901200, and 2019YFA0904900), the National Natural Science Foundation of China (31670041, 31970055), Shandong Provincial Funds for Distinguished Young Scientists (JQ 201806), Key R\&D Program of Shandong Provincial (2019GSF107034, 2019GSF107039) and Qilu Young Scholar of Shandong University. The funders had no role in study design, data collection and interpretation, or the decision to submit the work for publication. We also thank Dr. Zhifeng Li and Dr. Jingyao Qu from Core Facilities for Life and Environmental Sciences (State Key Laboratory of Microbial Technology, Shandong University) for assistance in mass spectrographic analysis.

\section{Author contributions}

C.G., C.M., and P.X. designed the research. Z.K., M.Z., K.G., Y.L., D.X., W.Z., and S.G. performed the research. Z.K. and C.G. analyzed the data. Z.K., C.G., C.M., and P.X. wrote the paper.

\section{Competing interests}

The authors declare that they have no conflict of interest.

\section{Additional information}

Any supplementary information and source data are available in the online version of the paper. Reprints and permissions information is available online at http://www.nature.com/reprints/index.html. Correspondence and requests for materials 
bioRxiv preprint doi: https://doi.org/10.1101/2020.07.07.187567; this version posted July 7, 2020. The copyright holder for this preprint (which was not certified by peer review) is the author/funder. All rights reserved. No reuse allowed without permission.

should be addressed to C.G. and P.X.. 
Figure 1 Regulation of L-2-HG catabolism by LhgR in P. putida W619. (a) Schematic

representation of genomic neighborhood analysis of $\operatorname{lhg} O$ in different bacteria. Orthologs are shown in the same color and the direction of gene transcription is indicated by arrows. CsiR, GntR family allosteric transcription factor regulating glutarate catabolism; CsiD, glutarate hydroxylase; LhgO, L-2-HG oxidase; GabD, succinate semialdehyde dehydrogenase; GabT, 4-aminobutyrate aminotransferase; GabP, 4-aminobutyrate transporter. (b, c) Growth of the derivatives of $P$. putida KT2440 in MSMs with glutarate (b) or L-2-HG (c) as the sole carbon source. Growth (closed symbols) and the consumption of carbon source (open symbols) of $P$. putida KT2440 ( $\Delta l h g O)$ harboring plasmid pME6032-lhgO (black lines with squares) and $P$. putida KT2440 ( $\Delta l h g O)$ harboring empty plasmid pME6032 (red lines with circles) were measured in MSMs containing $5 \mathrm{~g} \mathrm{~L}^{-1}$ glutarate (b) or L-2-HG (c) as the sole carbon source. (d) Schematic representation of the construction of pME6032-F2-lhgR-F1-lhgO and its transfer into $P$. putida KT2440 ( $\Delta c \operatorname{siR} \Delta l h g O)$ and $P$. putida KT2440 ( $\Delta c \operatorname{siR} \Delta \operatorname{csiD} \Delta l h g O)$ by electroporation. The deleted genes in P. putida KT2440 are indicated by dashed lines. The reaction catalyzed by $\mathrm{CsiD}$ is also demonstrated. (e) The activities of $\mathrm{LhgO}$ in $P$. putida KT2440 ( $\Delta c \operatorname{csiR} \Delta l h g O)$ and $P$. putida KT2440 ( $\Delta c \operatorname{csiR} \Delta c \operatorname{siD} \Delta l h g O)$ harboring either plasmid pME6032-F2-lhgR-F1-lhgO or empty plasmid pME6032 grown in MSM with glutarate, L-2HG, or D-2-HG as the sole carbon source. All data shown are means \pm standard deviations (s.d.) $(\mathrm{n}=3$ independent experiments). 
Figure 2 Purification and characterization of LhgR. (a) SDS-PAGE analysis of the purification of LhgR. Lane M, molecular weight markers; lane 1, crude extract of $E$. coli BL21(DE3) harboring pETDuet-lhgR; lane 2, purified His6-tagged LhgR using a HisTrap column. (b) Gel-filtration chromatography of the purified LhgR with the Superdex 200 10/300 GL column. Red curve, chromatogram of purified LhgR; Black line, standard curve for protein molecular mass standards. (c) LhgR can bind to the $\operatorname{lh} g O$ promoter region. F1 fragment containing the $\operatorname{lh} g O$ promoter region $(10 \mathrm{nM})$ was titrated by purified $\operatorname{LhgR}(0,20$, $40,60,80,100,120,140,160 \mathrm{nM}$ ). (d) DNase I footprinting analysis of LhgR binding to the $\operatorname{lhg} O$ promoter region. The $\mathrm{F} 1$ fragment was labeled with 6-carboxyfluorescein (FAM) and incubated with $2 \mu \mathrm{g}$ LhgR (blue line) or without LhgR (red line). The region protected by LhgR is indicated with a dotted box. (e) L-2-HG prevent LhgR binding to the $\operatorname{lhg} O$ promoter region. EMSAs were carried out with $\mathrm{F} 1$ fragment $(10 \mathrm{nM})$ and purified $\operatorname{LhgR}(60 \mathrm{nM})$ in the absence of any other tested compounds (0) and in the presence of $50 \mathrm{mM}$ different compounds. The leftmost lane without LhgR was used as the control.

\section{Figure 3 Design and optimization of the LHGFR. (a) Schematic representation of the} predicted conformational change of FRET-based L-2-HG biosensor LHGFR in the absence or presence of L-2-HG. (b) Dose-response curve of purified LHGFRoNoC for increasing concentrations (10 $\mathrm{nM}$ to $1 \mathrm{mM}$ ) of L-2-HG in $50 \mathrm{mM}$ Tris-HCl buffer (pH 7.4). The emission ratio of Venus to mTFP increased (430 nm excitation) after L-2-HG binding. (c) Heap map of the truncating the $\mathrm{N}$ - and $\mathrm{C}$-terminal amino acids of LhgR to $\Delta R_{\max }$. Color indicates the value of $\Delta R_{\max }$ and white indicates the untested variants. (d) Comparison of the 
$\Delta R_{\max }$ of a set of L-2-HG biosensor variants based on the C-terminal amino acid truncated of LhgR. (e, f) Evaluation of the specificity of the purified LHGFR $0 \mathrm{~N}_{3 \mathrm{C}}(\mathbf{e})$ and LHGFR $0 \mathrm{~N}_{\mathrm{C}}(\mathbf{f})$. The emission ratio changes of both biosensors were measured in the presence of $240 \mu \mathrm{M}$ of D-lactate, L-lactate, D-2-HG, or different intermediates of TCA cycle and L-lysine catabolism, respectively. The rightmost column in the presence of L-2-HG was used as the control. (g, h) Reversal of L-2-HG binding with LHGFR by conversion of L-2-HG to 2-KG. The emission ratio of purified LHGFRoN3C (g) and LHGFR0N7C (h) was recorded in the absence and presence of $20 \mu \mathrm{M}$ L-2-HG before and after the addition of $5 \mu \mathrm{M}$ purified $\mathrm{LhgO}$ for $10 \mathrm{~min}$. All data shown are means \pm s.d. $(\mathrm{n}=3$ independent experiments).

Figure 4 Validation of purified LHGFR for determination L-2-HG levels in body fluids and bacterial culture system. (a-d) Dose-response curves of purified LHGFR ${ }_{0 N 3 C}$ and LHGFR ${ }_{0 N 7 C}$ for increasing concentrations (10 $\mathrm{nM}$ to $2 \mathrm{mM})$ of $\mathrm{L}-2-\mathrm{HG}$ in serum $(\mathbf{a}, \mathbf{b})$ and urine (c, d). (e, f) Comparison between the quantitative results of L-2-HG in serum (e) and urine (f) by LCMS/MS and LHGFR. The gray circles and red circles represent the quantitative results of

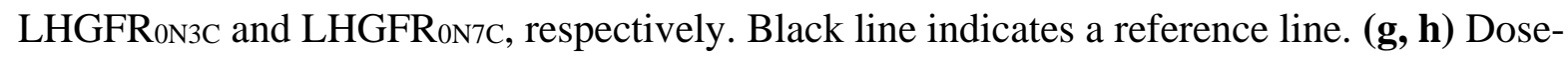
response curves of purified LHGFR $0 \mathrm{~N}_{3 \mathrm{C}}(\mathbf{g})$ and LHGFR $0 \mathrm{N7C}$ (h) for increasing concentrations (10 $\mathrm{nM}$ to $2 \mathrm{mM}$ ) of L-2-HG in bacterial culture medium. (i) Comparison between the quantitative results of L-2-HG in bacterial culture medium by LC-MS/MS and LHGFR. (j) Growth of $P$. putida KT2440 and its $l h g O$ mutant in medium containing $20 \mathrm{mM}$ glucose and $5 \mathrm{mM}$ glutarate as the carbon sources. $(\mathbf{k}, \mathbf{l})$ Determination of extracellular L-2-HG accumulation of $P$. putida KT2440 and its $\operatorname{lhg} O$ mutant by purified LHGFR 0 3C $(\mathbf{k})$ and 


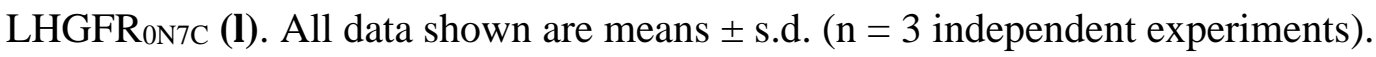

Figure 5 Monitoring L-2-HG fluctuations in living bacteria by LHGFR. (a) Time course of

the emission ratio changes of LHGFRoN3C expressed in E. coli BL21(DE3) in response to exogenous L-2-HG addition. All ratios were normalized to the control (ratio in the absence of L-2-HG at time point zero). (b) Normalized dose-response curve of LHGFR 0 3C expressed in E. coli BL21(DE3) for increasing concentrations (100 $\mathrm{nM}$ to $20 \mathrm{mM}$ ) of $\mathrm{L}-2-\mathrm{HG}$ at time point 60 min. (c) Time course of the emission ratio changes of LHGFR ${ }_{0 N 3 C}$ expressed in E. coli BL21(DE3) in response to the addition of $1 \mathrm{mM}$ glutarate, L-2-HG, D-2-HG, or glucose. All data were normalized to the control (ratio in the absence of any tested compounds at time point zero). (d) Detection of carbon starvation-induced L-2-HG accumulation over time by LHGFR $_{0 \mathrm{~N} 3 \mathrm{C}}$ expressed in E. coli $\mathrm{BL21}$ (DE3). Emission ratio changes of LHGFR ${ }_{0 \mathrm{~N} 3 \mathrm{C}}$ were measured when cultured in carbon starvation medium (black line) and medium with $20 \mathrm{mM}$ glucose (red line). All data were normalized to samples under carbon starvation condition at time point zero. (e) Long-term detection of L-2-HG fluctuations by LHGFR 0 N3C expressed in E. coli BL21(DE3). All data were normalized to samples under carbon starvation condition at time point zero. (f) Identification of the roles of CsiD and LhgO in endogenous L-2-HG catabolism during carbon starvation by LHGFR ${ }_{0 \mathrm{~N} 3 \mathrm{C}}$. Emission ratio changes of $\mathrm{LHGFR}_{0 \mathrm{~N} 3 \mathrm{C}}$ expressed in E. coli MG1655(DE3) wild-type (black line), E. coli MG1655(DE3) ( $\Delta c s i D)$ (red line), and E. coli MG1655(DE3) ( $\Delta l h g O)$ (blue line) were measured in carbon starvation medium. All data were normalized to time point zero of wild-type strain and shown as means \pm s.d. $(\mathrm{n}=3$ independent experiments). 
Figure 6 Monitoring L-2-HG fluctuations in human cells by LHGFR. (a) Confocal microscopy images of LHGFRon3C-expressing HEK293FT cells. The images are represented as mTFP channel, Venus channel, and overlay channel from left to right. Scale bar, $10 \mu \mathrm{m}$. (b) Normalized dose-response curve of LHGFR ${ }_{0 N 3 C}$ expressed in HEK293FT cells with increasing concentrations (10 nM to $10 \mathrm{mM}$ ) of L-2-HG. Cells were permeabilized with 10 $\mu \mathrm{M}$ digitonin. The emission ratio of non-permeabilized HEK293FT cells under physiological conditions is indicated with black dash line. (c) Responses of LHGFRoN3C expressed in HEK293FT cells to exogenously added $1 \mathrm{mM}$ glutarate, L-2-HG, D-2-HG, and glucose. All data were normalized to the control (ratio in the absence of any tested compounds). (d) Identification of the function of L2HGDH in L-2-HG catabolism by LHGFRon3c. The emission ratio was measured after co-transfecting siRNA targeting L2HGDH and LHGFRoN3C for 48 h. (e) Detection of hypoxia-induced L-2-HG accumulation by LHGFRoN3c. Emission ratio changes were recorded after LHGFRon3C-expressing HEK293FT cells cultured in normoxia or hypoxia in the absence and presence of $5 \mathrm{mM}$ dimethyl-2ketoglutarate $(\mathrm{DM} \alpha \mathrm{KG})$ for $24 \mathrm{~h}$. Emission ratio was normalized to normoxic condition without DMaKG. (f) Identification of the functions of LDHA and MDH2 in L-2-HG anabolism by LHGFRoN3c. HEK293FT cells were cultured in the presence of $5 \mathrm{mM}$ DMaKG. Emission ratio was normalized to normoxic condition treated with negative siRNA. All data shown are means \pm s.d. ( $(\mathrm{n}=3,3,4,4$, and 4 independent experiments for $\mathbf{b}, \mathbf{c}, \mathbf{d}, \mathbf{e}$, and $\mathbf{f})$. *, $P<0.05$ in two-tailed $t$ test; ${ }^{* *}, P<0.01$ in two-tailed $t$ test; ${ }^{* *}, P<0.001$ in two-tailed $t$ test; ns, no significant difference ( $P \geq 0.05$ in two-tailed $t$ test). 
a

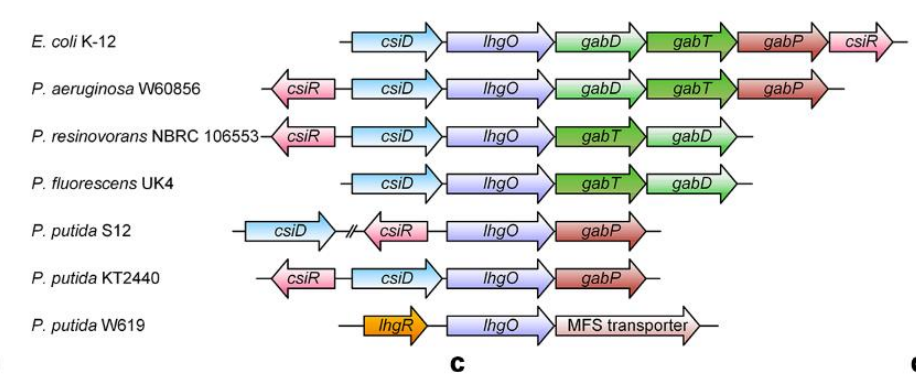

e

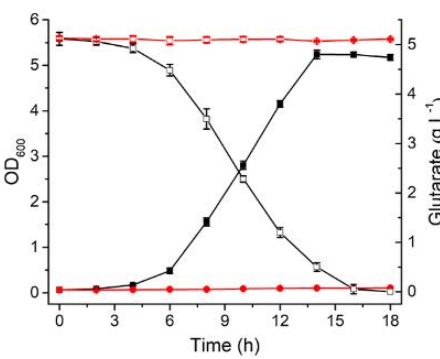

c

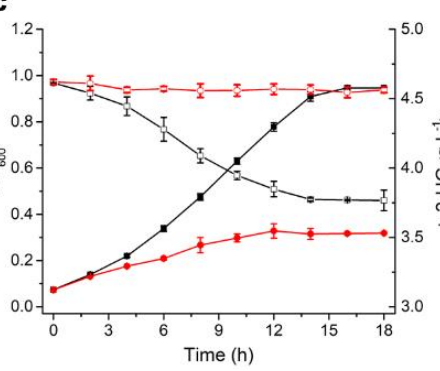

d

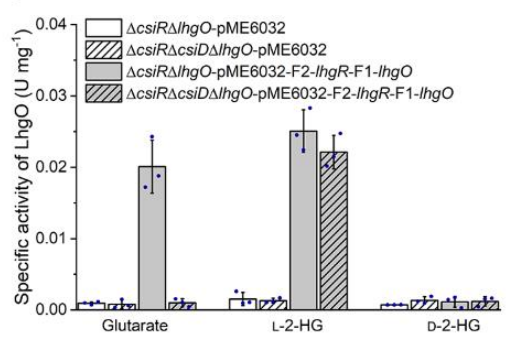

Pseudomonas putida W619

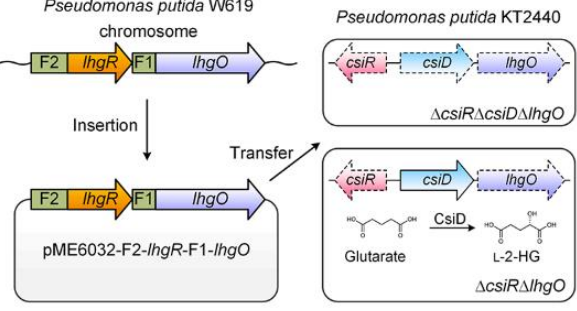

Figure 1 Regulation of L-2-HG catabolism by LhgR in P. putida W619. (a) Schematic representation of genomic neighborhood analysis of $\operatorname{lhg} O$ in different bacteria. Orthologs are shown in the same color and the direction of gene transcription is indicated by arrows. CsiR, GntR family allosteric transcription factor regulating glutarate catabolism; CsiD, glutarate hydroxylase; LhgO, L-2-HG oxidase; GabD, succinate semialdehyde dehydrogenase; GabT, 4-aminobutyrate aminotransferase; GabP, 4-aminobutyrate transporter. (b, c) Growth of the derivatives of $P$. putida KT2440 in MSMs with glutarate (b) or L-2-HG (c) as the sole carbon source. Growth (closed symbols) and the consumption of carbon source (open symbols) of $P$. putida KT2440 ( $\triangle l h g O)$ harboring plasmid pME6032-lhgO (black lines with squares) and P. putida KT2440 ( $\Delta l h g O)$ harboring empty plasmid pME6032 (red lines with circles) were measured in MSMs containing $5 \mathrm{~g} \mathrm{~L}^{-1}$ glutarate (b) or L-2-HG (c) as the sole carbon source. (d) Schematic representation of the construction of pME6032-F2-lhgR-F1-lhgO and its transfer into P. putida KT2440 $(\Delta c s i R \Delta l h g O)$ and $P$. putida KT2440 ( $\Delta c \operatorname{siR} \Delta c s i D \Delta l h g O)$ by electroporation. The deleted genes in $P$. putida KT2440 are indicated by dashed lines. The reaction catalyzed by CsiD is also demonstrated. (e) The activities of LhgO in $P$. putida KT2440 ( $\Delta c \operatorname{siR} \Delta l h g O)$ and $P$. putida KT2440 ( $\Delta c \operatorname{siR} \Delta c \operatorname{siD} \Delta l h g O)$ harboring either plasmid pME6032-F2-lhgR-F1-lhgO or empty plasmid pME6032 grown in MSM with glutarate, L-2-HG, or D-2-HG as the sole carbon source. All data shown are means \pm standard deviations (s.d.) ( $\mathrm{n}=3$ independent experiments). 
a

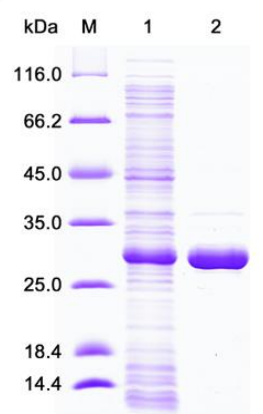

d

632 b
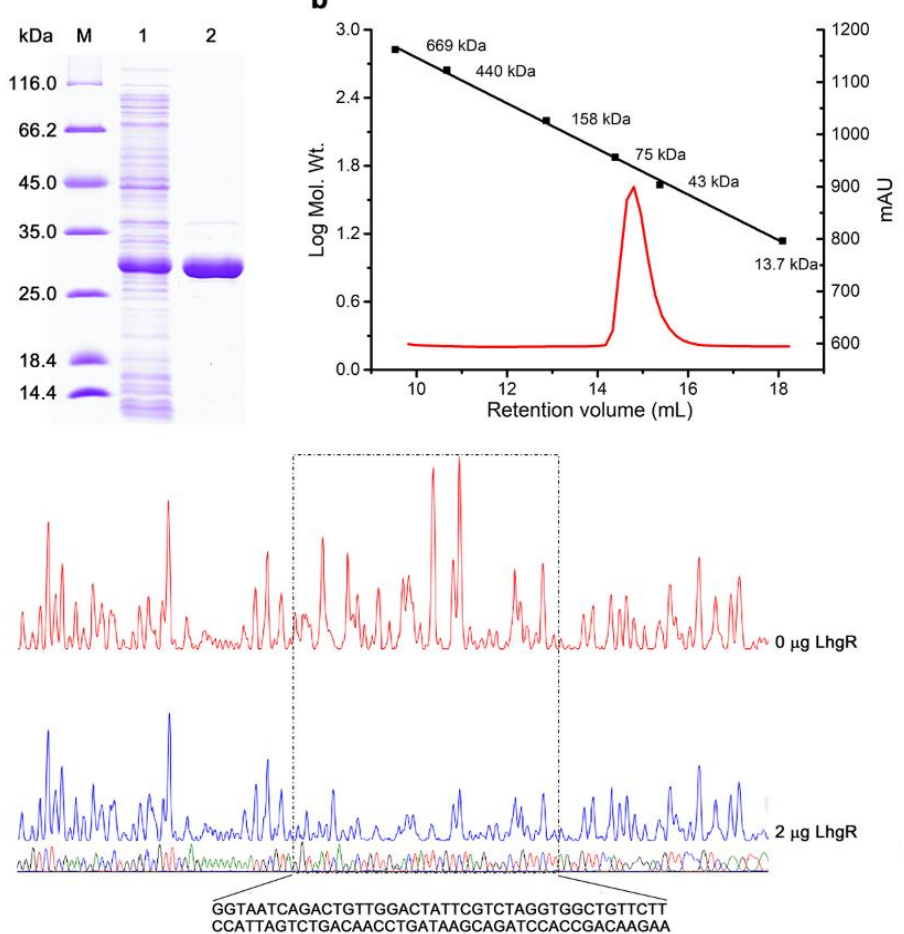

c
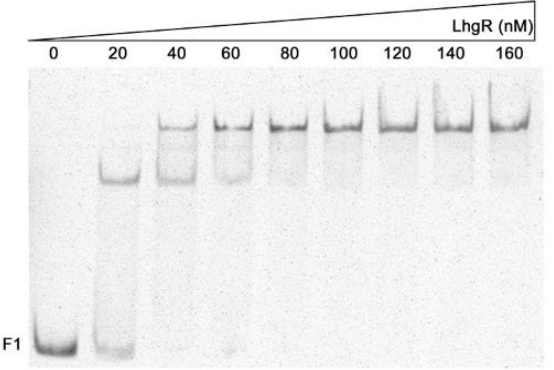

e

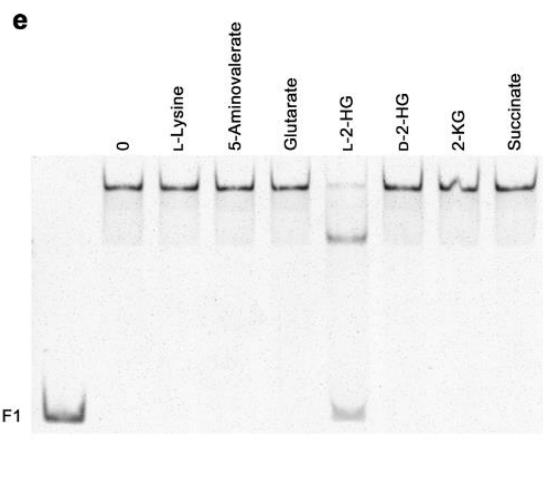

Figure 2 Purification and characterization of LhgR. (a) SDS-PAGE analysis of the purification of LhgR.

Lane M, molecular weight markers; lane 1, crude extract of E. coli BL21(DE3) harboring pETDuet-lhgR; lane 2, purified His6-tagged LhgR using a HisTrap column. (b) Gel-filtration chromatography of the purified LhgR with the Superdex 200 10/300 GL column. Red curve, chromatogram of purified LhgR;

Black line, standard curve for protein molecular mass standards. (c) LhgR can bind to the $\operatorname{lhg} O$ promoter region. F1 fragment containing the $l h g O$ promoter region $(10 \mathrm{nM})$ was titrated by purified $\operatorname{LhgR}(0,20,40$, $60,80,100,120,140,160 \mathrm{nM}$ ). (d) DNase I footprinting analysis of LhgR binding to the $\operatorname{lhg} O$ promoter region. The F1 fragment was labeled with 6-carboxyfluorescein (FAM) and incubated with $2 \mu \mathrm{g}$ LhgR (blue line) or without LhgR (red line). The region protected by LhgR is indicated with a dotted box. (e) L2-HG prevent LhgR binding to the $\operatorname{lhg} O$ promoter region. EMSAs were carried out with F1 fragment (10 $\mathrm{nM})$ and purified $\operatorname{LhgR}(60 \mathrm{nM})$ in the absence of any other tested compounds $(0)$ and in the presence of $50 \mathrm{mM}$ different compounds. The leftmost lane without LhgR was used as the control. 


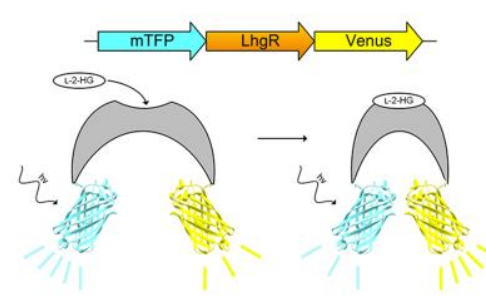

d

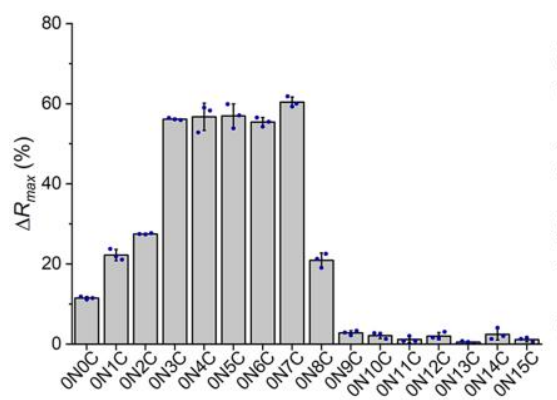

f

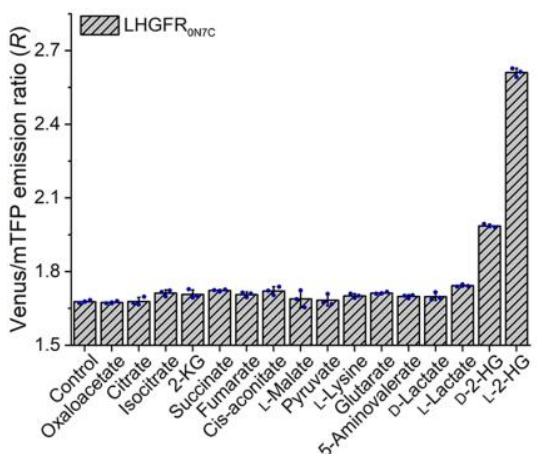

b
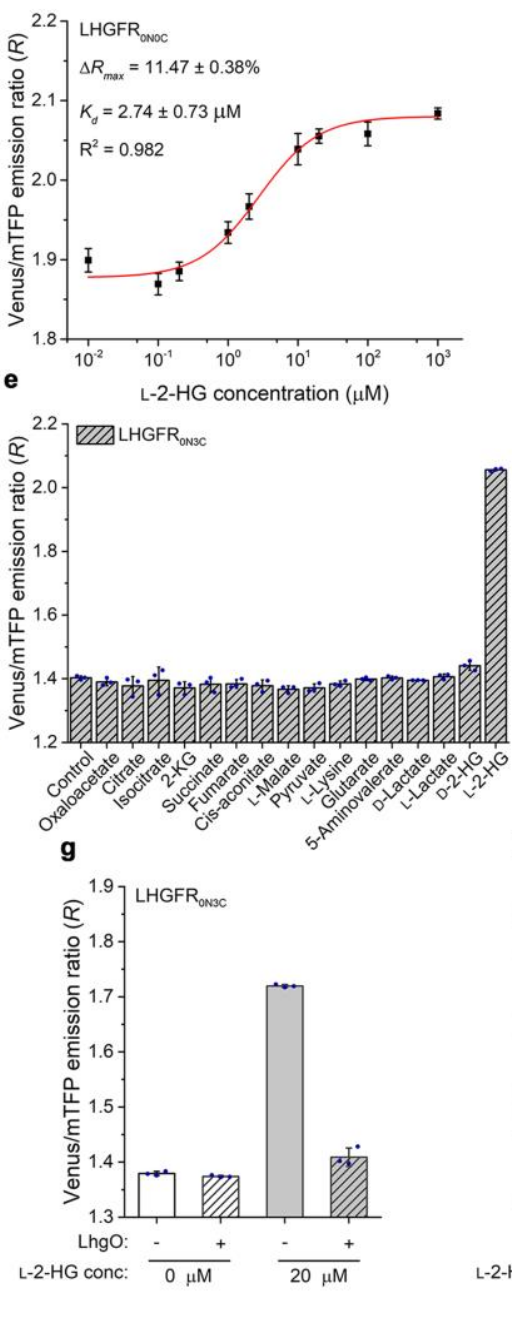

C
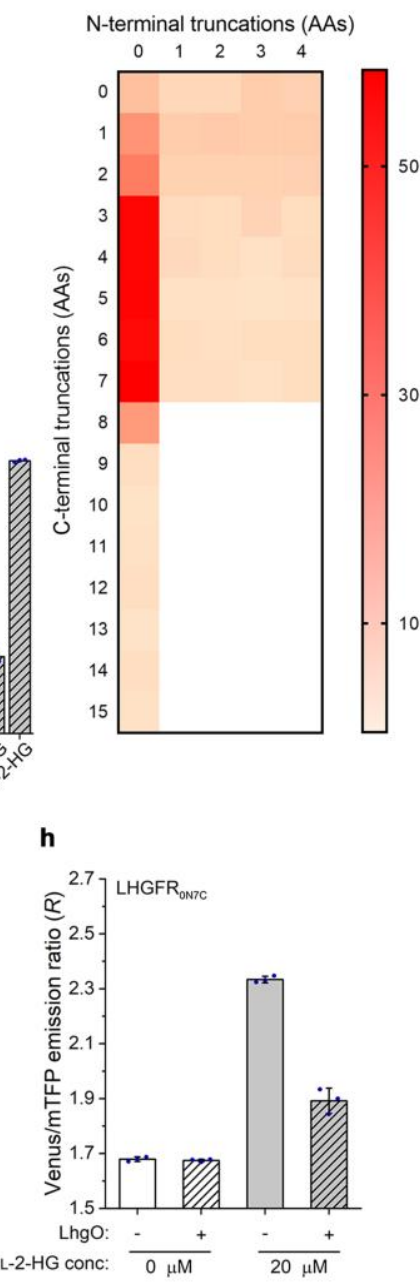

Figure 3 Design and optimization of the LHGFR. (a) Schematic representation of the predicted conformational change of FRET-based L-2-HG biosensor LHGFR in the absence or presence of L-2-HG.

(b) Dose-response curve of purified LHGFR ${ }_{0 \mathrm{~N} O \mathrm{C}}$ for increasing concentrations (10 $\mathrm{nM}$ to $1 \mathrm{mM}$ ) of L-2$\mathrm{HG}$ in $50 \mathrm{mM}$ Tris- $\mathrm{HCl}$ buffer (pH 7.4). The emission ratio of Venus to mTFP increased (430 nm excitation) after L-2-HG binding. (c) Heap map of the truncating the $\mathrm{N}$ - and C-terminal amino acids of LhgR to $\Delta R_{\max }$. Color indicates the value of $\Delta R_{\max }$ and white indicates the untested variants. (d) Comparison of the $\Delta R_{\max }$ of a set of L-2-HG biosensor variants based on the C-terminal amino acid truncated of LhgR. (e, f) Evaluation of the specificity of the purified LHGFR 0 3C $(\mathbf{e})$ and LHGFR 0 N7C $(\mathbf{f})$. The emission ratio changes of both biosensors were measured in the presence of $240 \mu \mathrm{M}$ of D-lactate, Llactate, D-2-HG, or different intermediates of TCA cycle and L-lysine catabolism, respectively. The rightmost column in the presence of L-2-HG was used as the control. (g, h) Reversal of L-2-HG binding with LHGFR by conversion of L-2-HG to $2-\mathrm{KG}$. The emission ratio of purified $\mathrm{LHGFR}_{0 \mathrm{~N} 3 \mathrm{C}}(\mathrm{g})$ and LHGFR $_{0 \mathrm{~N} 7 \mathrm{C}}(\mathbf{h})$ was recorded in the absence and presence of $20 \mu \mathrm{M}$ L-2-HG before and after the addition of $5 \mu \mathrm{M}$ purified $\mathrm{LhgO}$ for $10 \mathrm{~min}$. All data shown are means \pm s.d. ( $\mathrm{n}=3$ independent experiments). 

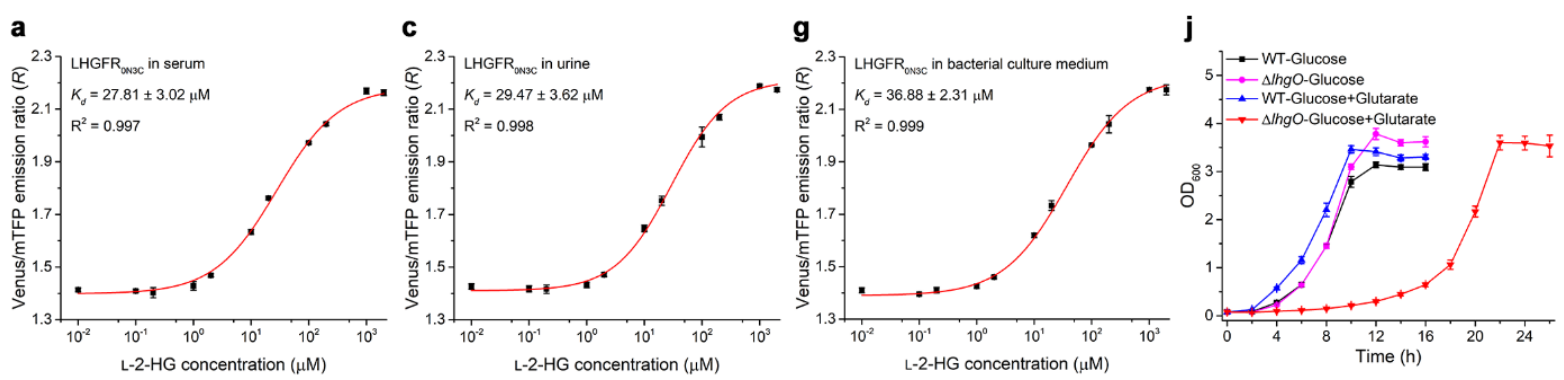

b

\section{d}
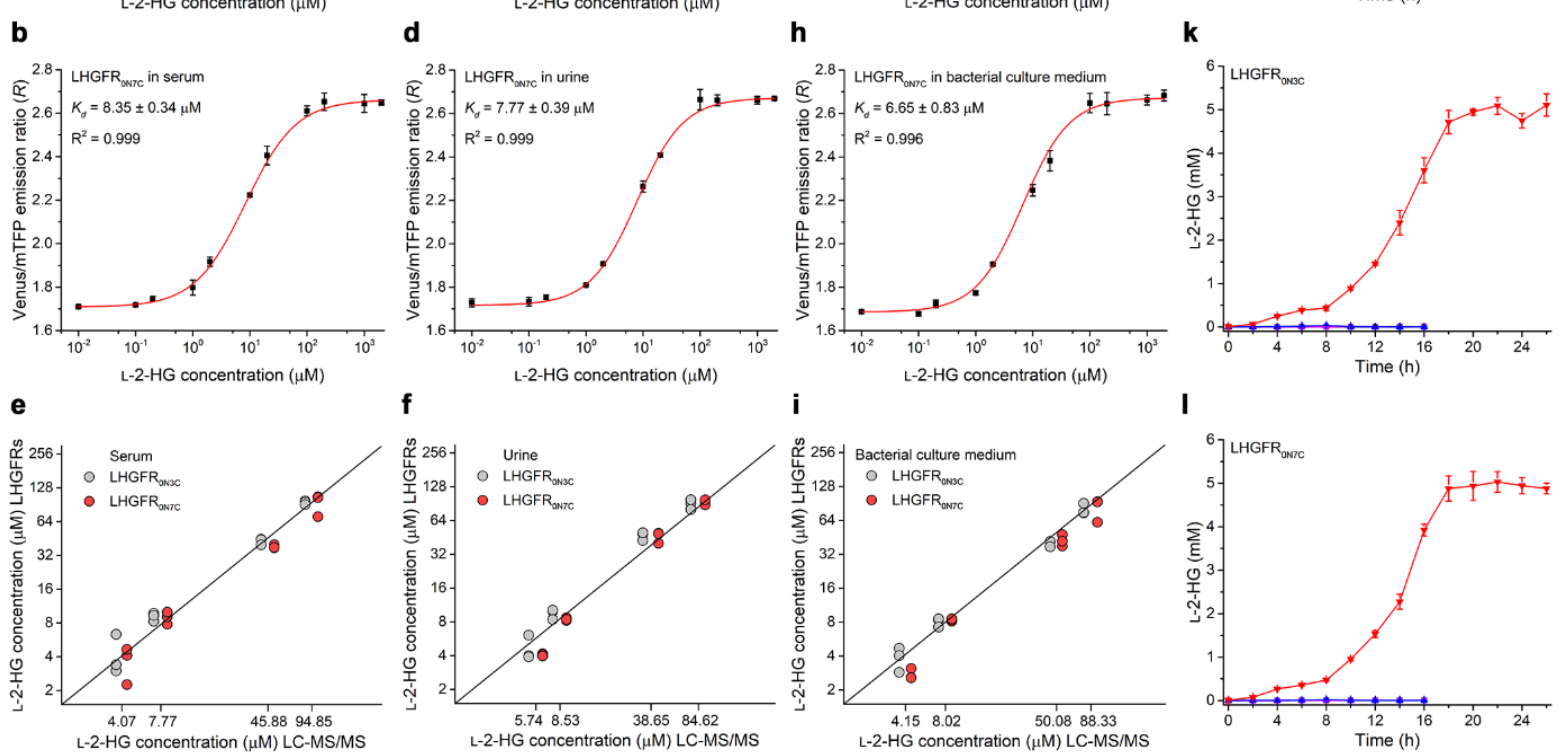

Figure 4 Validation of purified LHGFR for determination L-2-HG levels in body fluids and bacterial

culture system. (a-d) Dose-response curves of purified LHGFR NN3C $_{\text {and }}$ LHGFR $_{0 \mathrm{~N} 7 \mathrm{C}}$ for increasing concentrations (10 $\mathrm{nM}$ to $2 \mathrm{mM})$ of $\mathrm{L}-2-\mathrm{HG}$ in serum $(\mathbf{a}, \mathbf{b})$ and urine $(\mathbf{c}, \mathbf{d})$. (e, f) Comparison between the quantitative results of L-2-HG in serum (e) and urine (f) by LC-MS/MS and LHGFR. The gray circles and red circles represent the quantitative results of $\mathrm{LHGFR}_{0 \mathrm{~N} 3 \mathrm{C}}$ and $\mathrm{LHGFR}_{0 \mathrm{~N} 7 \mathrm{C}}$, respectively. Black line indicates a reference line. $(\mathbf{g}, \mathbf{h})$ Dose-response curves of purified LHGFR ${ }_{0 N 3 C}(\mathbf{g})$ and $\operatorname{LHGFR}_{0 \mathrm{~N} 7 \mathrm{C}}(\mathbf{h})$ for increasing concentrations (10 $\mathrm{nM}$ to $2 \mathrm{mM}$ ) of L-2-HG in bacterial culture medium. (i) Comparison between the quantitative results of L-2-HG in bacterial culture medium by LC-MS/MS and LHGFR. (j) Growth of $P$. putida KT2440 and its $l h g O$ mutant in medium containing $20 \mathrm{mM}$ glucose and $5 \mathrm{mM}$ glutarate as the carbon sources. $(\mathbf{k}, \mathbf{l})$ Determination of extracellular L-2-HG accumulation of $P$. putida KT2440 and its $\operatorname{lhg} O$ mutant by purified $\mathrm{LHGFR}_{0 \mathrm{~N} 3 \mathrm{C}}(\mathbf{k})$ and $\mathrm{LHGFR}_{0 \mathrm{~N} 7 \mathrm{C}}(\mathbf{l})$. All data shown are means \pm s.d. ( $n=3$ independent experiments). 


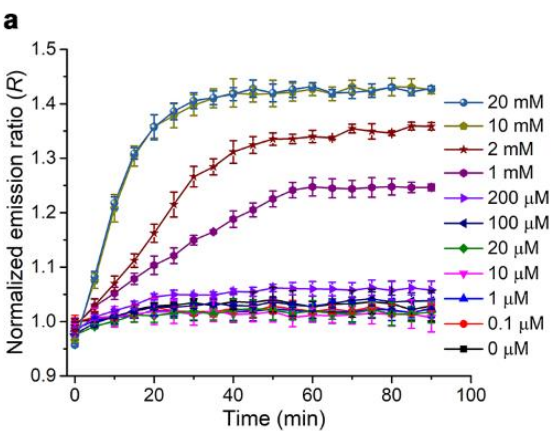

d

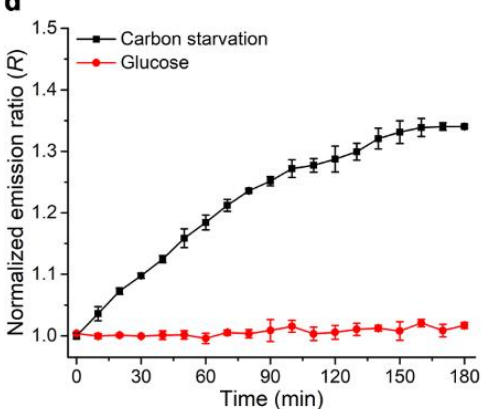

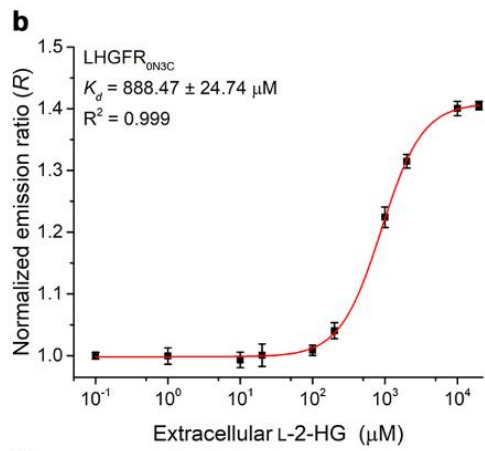

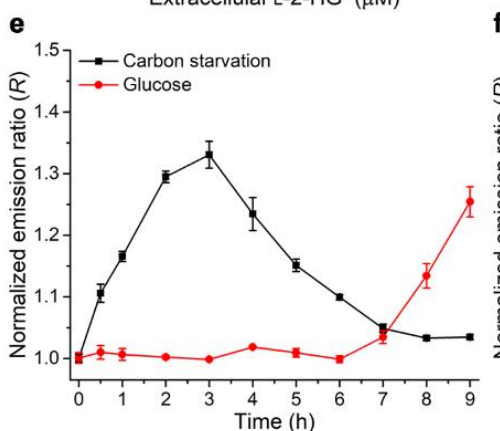

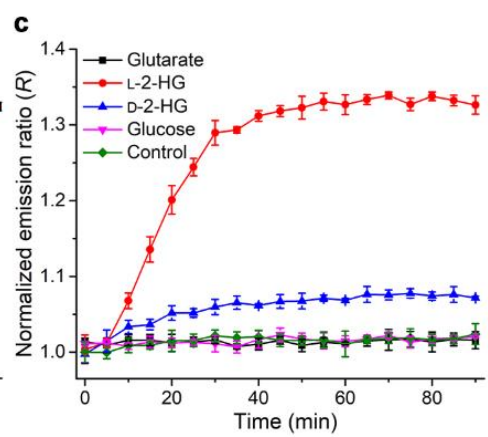

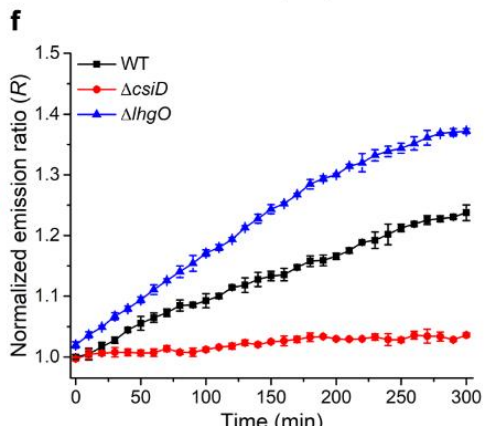

Figure 5 Monitoring L-2-HG fluctuations in living bacteria by LHGFR. (a) Time course of the emission ratio changes of $\mathrm{LHGFR}_{0 \mathrm{~N} 3 \mathrm{C}}$ expressed in E. coli BL21(DE3) in response to exogenous L-2-HG addition. All ratios were normalized to the control (ratio in the absence of L-2-HG at time point zero). (b) Normalized dose-response curve of LHGFR $_{0 \mathrm{~N} 3 \mathrm{C}}$ expressed in E. coli BL21(DE3) for increasing concentrations (100 nM to $20 \mathrm{mM}$ ) of L-2-HG at time point $60 \mathrm{~min}$. (c) Time course of the emission ratio changes of LHGFR $_{0 \mathrm{~N} 3 \mathrm{C}}$ expressed in E. coli BL21(DE3) in response to the addition of $1 \mathrm{mM}$ glutarate, L2-HG, D-2-HG, or glucose. All data were normalized to the control (ratio in the absence of any tested compounds at time point zero). (d) Detection of carbon starvation-induced L-2-HG accumulation over time by LHGFR $_{0 \mathrm{~N} 3 \mathrm{C}}$ expressed in $E$. coli $\mathrm{BL21}(\mathrm{DE} 3)$. Emission ratio changes of LHGFR $_{0 \mathrm{~N} 3 \mathrm{C}}$ were measured when cultured in carbon starvation medium (black line) and medium with $20 \mathrm{mM}$ glucose (red line). All data were normalized to samples under carbon starvation condition at time point zero. (e) Long-term detection of L-2-HG fluctuations by LHGFR ${ }_{0 \mathrm{~N} 3 \mathrm{C}}$ expressed in E. coli BL21(DE3). All data were normalized to samples under carbon starvation condition at time point zero. (f) Identification of the roles of CsiD and LhgO in endogenous L-2-HG catabolism during carbon starvation by LHGFR ${ }_{0 \mathrm{~N} 3 \mathrm{C}}$. Emission ratio changes of LHGFR $_{0 \mathrm{~N} 3 \mathrm{C}}$ expressed in E. coli MG1655(DE3) wild-type (black line), E. coli MG1655(DE3) ( $\Delta c s i D$ ) (red line), and E. coli MG1655(DE3) ( $\Delta l h g O)$ (blue line) were measured in carbon starvation medium. All data were normalized to time point zero of wild-type strain and shown as means \pm s.d. ( $\mathrm{n}=3$ independent experiments). 
a
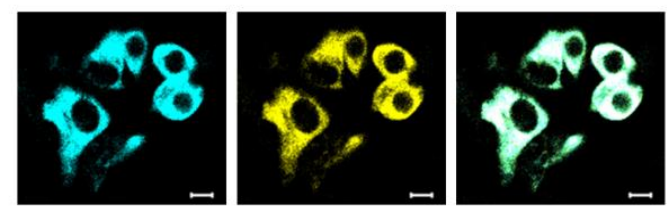

d

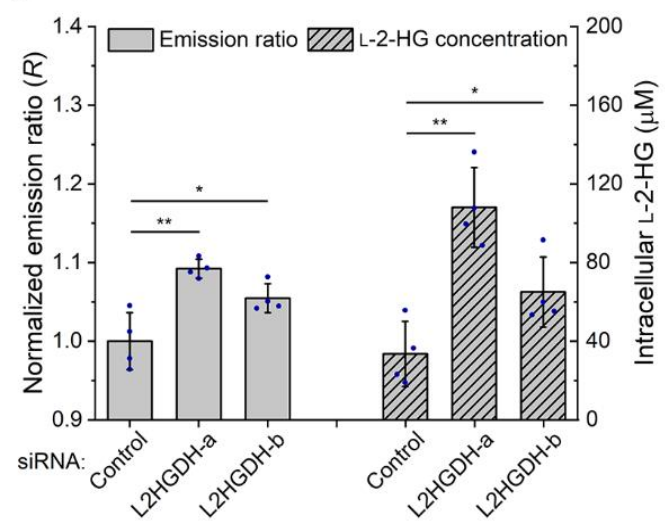

f

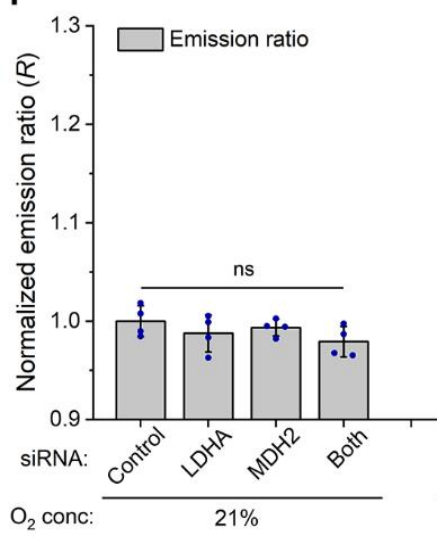

b

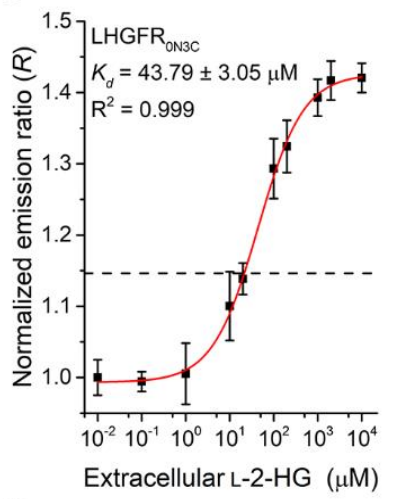

e

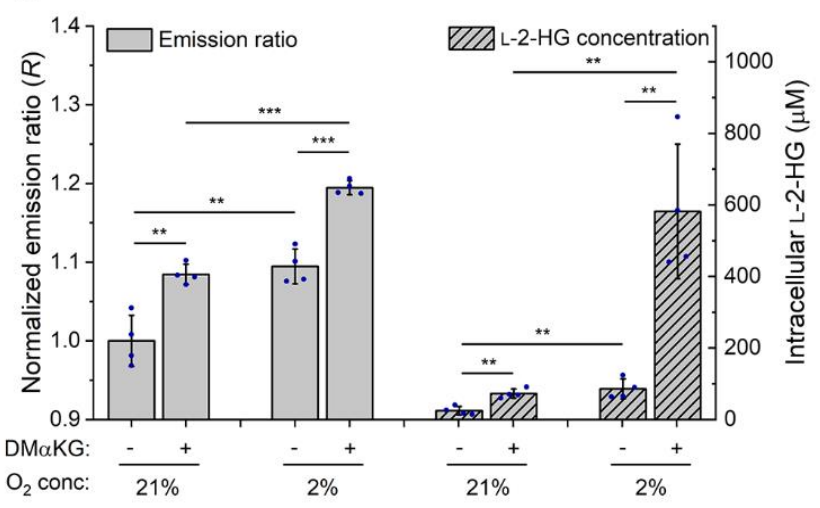

695

696

697

Figure 6 Monitoring L-2-HG fluctuations in human cells by LHGFR. (a) Confocal microscopy images of

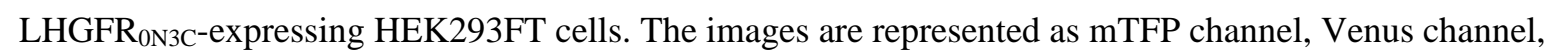
and overlay channel from left to right. Scale bar, $10 \mu \mathrm{m}$. (b) Normalized dose-response curve of LHGFR $_{0 \mathrm{~N} 3 \mathrm{C}}$ expressed in HEK293FT cells with increasing concentrations (10 $\mathrm{nM}$ to $10 \mathrm{mM}$ ) of L-2-HG. Cells were permeabilized with $10 \mu \mathrm{M}$ digitonin. The emission ratio of non-permeabilized HEK293FT cells under physiological conditions is indicated with black dash line. (c) Responses of LHGFR ${ }_{0 N 3 C}$ expressed in HEK293FT cells to exogenously added $1 \mathrm{mM}$ glutarate, L-2-HG, D-2-HG, and glucose. All data were normalized to the control (ratio in the absence of any tested compounds). (d) Identification of the function of L2HGDH in L-2-HG catabolism by LHGFR $_{0 \mathrm{~N} 3 \mathrm{C}}$. The emission ratio was measured after co-transfecting siRNA targeting L2HGDH and LHGFR ${ }_{0 N 3 C}$ for $48 \mathrm{~h}$. (e) Detection of hypoxia-induced L-2-HG accumulation by LHGFR $_{0 \mathrm{~N} 3 \mathrm{C}}$. Emission ratio changes were recorded after LHGFR $_{0 \mathrm{~N} 3 \mathrm{C}-\mathrm{expressing}}$ HEK293FT cells cultured in normoxia or hypoxia in the absence and presence of $5 \mathrm{mM}$ dimethyl-2- 
709 ketoglutarate $(\mathrm{DM} \alpha \mathrm{KG})$ for $24 \mathrm{~h}$. Emission ratio was normalized to normoxic condition without DMaKG.

710 (f) Identification of the functions of LDHA and MDH2 in L-2-HG anabolism by LHGFR 0 33. HEK293FT

711 cells were cultured in the presence of $5 \mathrm{mM}$ DM $\alpha \mathrm{KG}$. Emission ratio was normalized to normoxic

712 condition treated with negative siRNA. All data shown are means \pm s.d. $(\mathrm{n}=3,3,4,4$, and 4 independent

713 experiments for $\mathbf{b}, \mathbf{c}, \mathbf{d}, \mathbf{e}$, and $\mathbf{f}) .{ }^{*}, P<0.05$ in two-tailed $t$ test; ${ }^{* *}, P<0.01$ in two-tailed $t$ test; ${ }^{* * *}, P<$

$714 \quad 0.001$ in two-tailed $t$ test; ns, no significant difference ( $P \geq 0.05$ in two-tailed $t$ test). 


\section{Online methods}

\section{Bacterial strains and culture conditions}

The bacterial strains used in this study are listed in Supplementary Table 3. E. coli and its derivatives were cultured in Luria-Bertani (LB) broth at $37^{\circ} \mathrm{C}$ and $180 \mathrm{rpm}$. P. putida KT2440 and its derivatives were grown in minimal salt mediums (MSMs) containing different carbon sources at $30{ }^{\circ} \mathrm{C}$ and $200 \mathrm{rpm}$. Antibiotics were used at the following concentrations: tetracycline at $30 \mu \mathrm{g} \mathrm{mL} L^{-1}$; kanamycin at $50 \mu \mathrm{g} \mathrm{mL}^{-1}$; ampicillin at $100 \mu \mathrm{g}$ $\mathrm{mL}^{-1}$; spectinomycin, at $50 \mu \mathrm{g} \mathrm{mL} L^{-1}$; and chloramphenicol at $40 \mu \mathrm{g} \mathrm{mL} \mathrm{m}^{-1}$.

\section{Cloning of F2-lhgR-F1-lhgO and lhgO}

All the plasmids and primers used in this study are listed in Supplementary Table 3 and Table 4, respectively. The gene segment F2-lhgR-F1-lhg $O$ of $P$. putida W619 was synthesized by Tongyong Biosystem Co., Ltd (China). The $\operatorname{lhg} O$ gene of $P$. putida W619 was amplified and cloned into pME6032 plasmid using the restriction sites of EcoRI and KpnI to construct pME6032-lhgO and the $P_{\text {tac }}$ promoter of pME6032 was replaced by the gene segment F2lhgR-F1-lhgO using the restriction sites of SacI and BamHI to construct pME6032-F2-lhgRF1-lhg $O$, then both recombinant plasmids was transferred into different derivatives of $P$. putida KT2440 by electroporation, respectively.

\section{Construction of $P$. putida KT2440 and $E$. coli MG1655(DE3) mutants}

Genes of $P$. putida KT2440 were deleted via allele exchange using the pK18mobsacB system $^{49}$. Briefly, the homologous arms upstream and downstream of the target gene were PCR amplified and fused together by recombinant PCR. The generated fusion fragment was cloned into the suicide plasmid pK18mobsacB. The resulting plasmid was transferred into $P$. 
putida KT2440 by electroporation. The single crossover cells and the second crossover cells were sequentially screened from LB plates containing $50 \mu \mathrm{g} \mathrm{mL}{ }^{-1}$ kanamycin or $10 \%$ (wt/vol) sucrose, respectively.

To construct the E. coli MG1655(DE3) ( $\triangle c s i D$ ) mutant strain, the homologous arm upstream of the $c s i D$ gene, kanamycin resistance cassette, and the homologous arm downstream of the $c s i D$ gene were PCR amplified using the primers $c s i D-\mathrm{F} 1 / c s i D-\mathrm{R} 1, c s i D$ F2/csiD-R2, and $c s i D-F 3 / c s i D-\mathrm{R} 3$, respectively. The PCR products were fused together by recombinant PCR, and the resulting fusion was transferred into E. coli MG1655(DE3) harboring pTKRed plasmid following isopropyl- $\beta$-D-1-thiogalactopyranoside (IPTG) induction. The recombinant cells were selected on LB plates containing $50 \mu \mathrm{gLL}^{-1}$ kanamycin at $37^{\circ} \mathrm{C}$. The pCP20 plasmid was transferred into the selected cells, followed by a second screening on LB plates containing $40 \mu \mathrm{g} \mathrm{mL} L^{-1}$ chloramphenicol at $30{ }^{\circ} \mathrm{C}$, then cultured in LB medium at $42{ }^{\circ} \mathrm{C}$ to eliminate pCP20 plasmid. The $l h g O$ mutant of $E$. coli MG1655(DE3) were generated by the same process. All mutants were verified by PCR and sequencing.

\section{Enzymatic assay of LhgO}

The derivatives of $P$. putida KT2440 were cultured in $50 \mathrm{~mL}$ MSMs with $5 \mathrm{~g} \mathrm{~L}^{-1}$ different compounds as carbon sources at $30^{\circ} \mathrm{C}$ and $200 \mathrm{rpm}$. The cells were harvested at mid-log phase, washed twice and resuspended in phosphate-buffered saline (PBS), then lysed by sonication on ice after the addition of $1 \mathrm{mM}$ phenylmethylsulfonyl fluoride (PMSF). The supernatants obtained were used for further enzyme activities measurements after a centrifugation process $\left(13,000 \times g\right.$ for $10 \mathrm{~min}$ at $\left.4{ }^{\circ} \mathrm{C}\right)$. Protein concentrations of the 
supernatants were determined using the Bradford protein assay kit (Sangon, China).

The activity of $\mathrm{LhgO}$ was assayed at $30^{\circ} \mathrm{C}$ by monitored the reduction of dichlorophenol-indophenol (DCPIP) corresponding to the change of absorbance at $600 \mathrm{~nm}$ using a UV/visible spectrophotometer (Ultrospec 2100 pro, Amersham Biosciences, USA). The $800 \mu \mathrm{L}$ reaction solution contained $0.1 \mathrm{mM} \mathrm{L-2-HG}, 0.05 \mathrm{mM}$ DCPIP, $0.2 \mathrm{mM}$ phenazine methosulfate (PMS) in PBS and $40 \mu \mathrm{L}$ crude extracts. One unit of LhgO activity was defined as the amount of enzyme that catalyzed the reduction of $1 \mu \mathrm{mol}$ of DCPIP per minute.

\section{Expression, purification, and characterization of $\operatorname{LhgR}$}

To express and purify the recombinant LhgR, the $l h g R$ gene was PCR amplified using the primer pair $\operatorname{lhg} R-\mathrm{F} / \operatorname{lhg} R-\mathrm{R}$, which contained BamHI and HindIII restriction sites, respectively, and then cloned into the pETDuet-1 plasmid to construct pETDuet-lhgR. The $E$. coli BL21(DE3) strains harboring pETDuet-lhgR plasmid were grown to an OD600 of 0.6 in $\mathrm{LB}$ medium at $37^{\circ} \mathrm{C}$, after which the cells were induced for $12 \mathrm{~h}$ with $1 \mathrm{mM} \mathrm{IPTG}$ at $16{ }^{\circ} \mathrm{C}$. The cells were harvested, washed twice and resuspended in buffer A ( $20 \mathrm{mM}$ sodium phosphate and $500 \mathrm{mM}$ sodium chloride, $\mathrm{pH}$ 7.4), then lysed by sonication on ice after addition of $1 \mathrm{mM}$ PMSF and 10\% (vol/vol) glycerol. The cell lysate was centrifuged at $13,000 \times \mathrm{g}$ for 40 min at $4{ }^{\circ} \mathrm{C}$, and the resultant supernatant was loaded onto a HisTrap HP column ( $5 \mathrm{~mL})$ equilibrated with buffer A. The target protein was eluted with buffer B (20 $\mathrm{mM}$ sodium phosphate, $500 \mathrm{mM}$ sodium chloride, and $500 \mathrm{mM}$ imidazole, $\mathrm{pH}$ 7.4), analyzed by $12.5 \%$ sodium dodecyl sulfate-polyacrylamide gel electrophoresis (SDS-PAGE), quantified by the Bradford protein assay kit (Sangon, China). 
To determine the native molecular weight of LhgR, gel-filtration chromatography was performed using a Superdex 200 10/300 GL column (GE Healthcare, Germany) and standard proteins including thyroglobulin $(669 \mathrm{kDa})$, ferritin $(440 \mathrm{kDa})$, aldolase $(158 \mathrm{kDa})$, conalbumin $(75 \mathrm{kDa})$, ovalbumin $(43 \mathrm{kDa})$, and ribonuclease $\mathrm{A}(13.7 \mathrm{kDa})$. The eluent buffer contained $50 \mathrm{mM}$ sodium phosphate and $150 \mathrm{mM}$ sodium chloride (pH 7.2).

\section{Electrophoretic mobility shift assays}

Electrophoretic mobility shift assays (EMSAs) were carried out using the DNA fragment (F1 or F2) and purified LhgR. The DNA fragments were first amplified by primer pairs F1-F/F1incubated with LhgR (0-160 nM) in $20 \mu \mathrm{L}$ EMSA binding buffer (10 mM Tris-HCl [pH 7.4], $50 \mathrm{mM} \mathrm{KCl}, 0.5 \mathrm{mM}$ EDTA, 10\% [vol/vol] glycerol, and 1mM dithiothreitol [DTT]). The binding reactions were carried out at $30^{\circ} \mathrm{C}$ for $30 \mathrm{~min}$. Electrophoresis was performed on $6 \%$ native polyacrylamide gels at $4{ }^{\circ} \mathrm{C}$ and $170 \mathrm{~V}$ (constant voltage) for about $45 \mathrm{~min}$, followed by staining with SYBR green I (TaKaRa, China) and photographing. probe and purified LhgR. The DNA fragment F1 were PCR amplified using the primer pair 
Cloning Kit (TransGen, China). The FAM-labeled probes were PCR amplified using the resulting plasmid and the primer pair M13F-FAM/M13R. Then, 350 ng probes were incubated with $2 \mu \mathrm{g}$ purified $\mathrm{LhgR}$ in a total volume of $40 \mu \mathrm{L}$ for $30 \mathrm{~min}$ at $30^{\circ} \mathrm{C}$. The DNase I digestion reaction was carried out by adding a total volume of $10 \mu \mathrm{L}$ solution containing approximately 0.015 units of DNase I (Promega, USA) and $100 \mathrm{nmol} \mathrm{CaCl}_{2}$ and further incubating for $1 \mathrm{~min}$ at $37^{\circ} \mathrm{C}$, then stopped by adding a total volume of $140 \mu \mathrm{L}$ stop solution containing $0.15 \%$ (wt/vol) SDS, $200 \mathrm{mM}$ unbuffered sodium acetate, and $30 \mathrm{mM}$ EDTA. The digested DNA fragments were first extracted with phenol-chloroform, then precipitated with ethanol and resuspended in $30 \mu \mathrm{L}$ MiliQ water. The binding region of LhgR to DNA fragment F2 was analyzed using the same procedure.

\section{Construction and purification of LHGFR}

The genes encoding mTFP and Venus were synthesized by Tongyong Biosystem Co., Ltd (China). The mTFP gene and Venus gene were amplified and cloned into pETDuet-1 plasmid using the BamHI and SacI restriction sites, and SalI and NotI restriction sites, respectively. Then either the full-length $l h g R$ gene, its truncated variants, or variants with artificial linkers were inserted between mTFP and Venus by the T5 exonuclease DNA assembly (TEDA) method $^{50}$, respectively. For expression in HEK293FT cells, the codon-optimized LHGFR 0 N3C or LHGFR 0 N7C sequence was synthesized and cloned into pcDNA3. $1^{(+)}$plasmid behind a Kozak sequence, 5'-GCCACC-3'. The L-2-HG biosensor LHGFR and its derivatives were expressed and purified using the same procedure.

\section{Characterization of LHGFR in vitro}

Purified L-2-HG biosensors and different compounds were diluted by $50 \mathrm{mM}$ Tris- $\mathrm{HCl}$ buffer 
$(\mathrm{pH} 7.4)$, mixed together in a black 96-well plate at a volume ratio of $3: 1$, and the

fluorescence intensities were measured using an EnSight microplate reader (PerkinElmer,

USA) with excitation at $430 \mathrm{~nm}$, emission at $485 \mathrm{~nm}$ (mTFP) and $528 \mathrm{~nm}$ (Venus). The doseresponse curves were fitted by OriginPro 2016 software (OriginLab) according to the following formula:

$$
R=R_{\max }+\frac{R_{\min }-R_{\max }}{1+\left([\mathrm{L}-2-\mathrm{HG}] / K_{d}\right)^{p}}
$$

where $R, R_{\max }$, and $R_{\min }$ refer to the emission ratio of Venus to mTFP, ratio in the absence of L-2-HG, and ratio at saturation with L-2-HG, respectively. The [L-2-HG], $K_{d}$, and $p$ refer to the L-2-HG concentration, apparent dissociation constant, and Hill slope, respectively. Emission spectra were recorded at $430 \mathrm{~nm}$ excitation, in steps of $2 \mathrm{~nm}$. The reversibility of LHGFR was determined by recording the emission ratios 10 min after the addition of $5 \mu \mathrm{M}$ purified LhgO, the control test without addition of L-2-HG or purified LhgO was run in parallel. The $\mathrm{pH}$ stability of LHGFR was determined using $50 \mathrm{mM}$ Tris-HCl buffer with $\mathrm{pH}$ adjusted from 4.0 to 9.0. The background fluorescence without the addition of LHGFR was subtracted.

In order to test the ability of LHGFR for quantitative analysis L-2-HG in different biological samples, purified LHGFR was diluted by $50 \mathrm{mM}$ Tris- $\mathrm{HCl}(\mathrm{pH}$ 7.4), while varying concentrations of L-2-HG was added into the serum and urine of healthy adults and bacteria culture medium and filtered through a $0.22 \mu \mathrm{m}$ filter, respectively. The mixtures were then incubated in a black 96-well plate at a volume ratio of 3:1, and the emission ratios were determined. The background fluorescence without the addition of LHGFR was subtracted.

\section{Quantification of L-2-HG by HPLC and LC-MS/MS}


When L-2-HG was used as carbon source to cultivate $P$. putida KT2440, its consumption was analyzed by using high-performance liquid chromatography (HPLC) system (Agilent 1100 series, Agilent Technologies, USA) equipped with an Aminex HPX-87H column $(300 \times 7.8$ mm, Bio-Rad, USA) and a RID detector at $55^{\circ} \mathrm{C}$ as described previously ${ }^{7}$.

To detect L-2-HG concentrations in various biological samples by liquid chromatography-tandem mass spectrometry (LC-MS/MS) system, the samples containing D,L-2-hydroxyglutarate disodium salt (2,3,3-D3) as internal standard (ITSD) were centrifuged at $13,000 \times g$ for $15 \mathrm{~min}$, then filtered through a $0.22 \mu \mathrm{m}$ filter. The serum samples were mixed with methanol at a volume ratio of 1:3 and vortexed for 2 min to remove protein before centrifugation. Samples were analyzed by using a Thermo ultimate 3000 rapid separation liquid chromatograph system (ThermoFisher, USA) coupled with a Bruker impact HD ESI-Q-TOF mass spectrometer (Bruker Daltonics, Germany) in negative ion mode and equipped with a Chirobiotic R column $(250 \times 4.6 \mathrm{~mm}$, Supelco Analytical, USA). Mobile phase was prepared from (A) $0.1 \%$ triethylamine adjusted to $\mathrm{pH} 4.5$ with acetic acid or (B) methanol. The quantification was conducted with an injection volume of $20 \mu \mathrm{L}$, a constant $5 \%$ gradient of (B) at a flow rate of $0.5 \mathrm{~mL} \mathrm{~min}^{-1}$, and a total analysis time of $15 \mathrm{~min}$.

\section{Characterization of LHGFR in living bacteria}

E. coli BL21(DE3) strains harboring either pETDuet-LHGFRon3C or pETDuet-LHGFRoN7C were grown to an $\mathrm{OD} 600$ of 0.6 in $\mathrm{LB}$ medium at $37{ }^{\circ} \mathrm{C}$, after which the cells were induced overnight in the presence of $1 \mathrm{mM} \mathrm{IPTG}$ at $16^{\circ} \mathrm{C}$. The cultures were collected by centrifugation at $6000 \times \mathrm{g}$ for $5 \mathrm{~min}$, washed three times, and resuspended to an $\mathrm{OD}_{600}$ of 2.5 by carbon starvation medium (MSM containing no carbon source) or glucose medium (MSM 
containing $20 \mathrm{mM}$ glucose).

To characterize the sensitivity and specificity of LHGFR $0 \mathrm{~N}_{3 \mathrm{C}}$ and LHGFRoN7c expressed in $E$. coli $\mathrm{BL} 21$ (DE3), $90 \mu \mathrm{L}$ cell suspensions following $8 \mathrm{~h}$ carbon starvation were mixed with $10 \mu \mathrm{L}$ increasing concentrations of L-2-HG or other compounds, and then added into a black 96-well plate (total $100 \mu \mathrm{L} /$ per well), the fluorescence intensities were determined using an EnSight microplate reader (PerkinElmer, USA) and the following instrument settings: excitation at $430 \mathrm{~nm}$, emission at $485 \mathrm{~nm}$ (mTFP) and $528 \mathrm{~nm}$ (Venus), time intervals of $5 \mathrm{~min}$, temperature at $37^{\circ} \mathrm{C}$, and shake at $180 \mathrm{rpm}$. For carbon starvation experiments, cell suspensions in carbon starvation medium or glucose medium were added into a black 96-well plate (100 $\mu \mathrm{L} /$ per well), then the fluorescence intensities were monitored every ten minutes. In order to analyze functions of $\mathrm{CsiD}$ and $\mathrm{LhgO}$ in endogenous L-2-HG anabolism and catabolism during carbon starvation, pETDuet-LHGFRon3C or pETDuetLHGFRon7c was transferred into E. coli MG1655(DE3) and its variants, and the assays were performed using the same procedure. The background fluorescence from wells containing $E$. coli cultures harboring pETDuet-1 plasmid was subtracted at each emission wavelength.

\section{Cell culture and live-cell imaging}

HEK293FT cells were cultured in high-glucose Dulbecco's modified eagle medium (DMEM) supplemented with $10 \%$ (vol/vol) fetal bovine serum (FBS), $100 \mu$ nits $\mathrm{mL}^{-1}$ penicillin, and $100 \mu \mathrm{g} \mathrm{mL} \mathrm{m}^{-1}$ streptomycin (all purchased from ThermoFisher, USA), and kept at $37^{\circ} \mathrm{C}$ in humidified air containing $5 \% \mathrm{CO}_{2}$. For hypoxia experiments, cells were kept in a compact $\mathrm{O}_{2}$ and $\mathrm{CO}_{2}$ subchamber controller (ProOx C21, BioSpherix, USA) at $2 \% \mathrm{O}_{2}, 5 \% \mathrm{CO}_{2}$, and balanced with $\mathrm{N}_{2}$ for $24 \mathrm{~h}$. For construction of LHGFR expressing cell, HEK293FT cells 
were plated in 24-well plates and transfected with pcDNA3. $1^{(+)}$plasmid encoding either LHGFRoN3C or LHGFRoN7C.

Live-cell imaging was carried out using a Zeiss 800 confocal microscope $48 \mathrm{~h}$ following transfection. LHGFRoN3C or LHGFRoN7c expressed in HEK293FT cells was excited using a $405 \mathrm{~nm}$ laser, and the emission was divided into a 460-500 nm channel (mTFP) and a 500550 channel (Venus).

\section{Characterization of LHGFR in HEK293FT cells}

To characterize the sensitivity and specificity of LHGFR expressed in HEK293FT cells, cells were trypsinized $48 \mathrm{~h}$ following transfection and suspended in $1 \times$ Hank's balanced salt solution supplemented with $20 \mathrm{mM}$ HEPES. Increasing concentrations of L-2-HG or other compounds including glutarate, D-2-HG, and glucose was mixed with the cell suspensions in a 96-well plate, respectively. Digitonin at a concentration of $10 \mu \mathrm{M}$ was used to induce cell permeabilization and deplete intracellular L-2-HG for in vivo response curves construction. Then, the fluorescence intensities were determined by a SpectraMax i3 fluorescence plate reader (Molecular Devices, USA) with excitation at $430 \mathrm{~nm}$ and emission at $485 \mathrm{~nm}$ (mTFP) and $528 \mathrm{~nm}$ (Venus). Basal L-2-HG concentration in HEK293FT cells under physiological conditions was determined by substituting the emission ratios of non-permeabilized HEK293FT cells into the calibrated in vivo response curves.

For the detection of hypoxia-induced production of L-2-HG, LHGFRoN3C was expressed in HEK293FT cells and cultured for $24 \mathrm{~h}$ at $2 \%$ oxygen or $21 \%$ oxygen in the absence or presence of $5 \mathrm{mM}$ dimethyl-2-ketoglutarate (DMaKG). The preparation of cell suspensions and the measurement of emission ratios were performed using the same procedure. The 
background fluorescence was subtracted at each emission wavelength. SiRNA experiments

The following Silencer Select siRNAs used in this study were purchased from ThermoFisher

Scientific (USA): negative control (4390846), L2HGDH-a (s36692), L2HGDH-b (s36693),

LDHA (s351), and MDH2 (s8622). To analyze L2HGDH functions in L-2-HG catabolism, siRNA targeting L2HGDH and pcDNA3. $1^{(+)}$plasmid encoding either LHGFR 0 N3C or LHGFR0N7C were mixed with Lipofectamine 3000 Transfection Reagent (ThermoFisher, USA) in Opti-MEM Reduced Serum Medium, and the lipoplexes prepared were transfected into HEK293FT cells according to the manufacturer's protocol. The fluorescence intensities were measured by a SpectraMax i3 fluorescence plate reader $48 \mathrm{~h}$ following transfection. Similarly, HEK293FT cells were transfected by LHGFRoN3C and siRNAs targeting LDHA and $\mathrm{MDH} 2$ separately or in combination. After transfection, cells were cultured sequentially under normoxic condition for $24 \mathrm{~h}$ and hypoxic condition for $24 \mathrm{~h}$ in the presence of $5 \mathrm{mM}$ $\mathrm{DM} \alpha \mathrm{KG}$, then the fluorescence intensities were measured. The cells cultured under normoxic condition in the presence of $5 \mathrm{mM} \mathrm{DM \alpha KG}$ for $48 \mathrm{~h}$ were set as control. The background fluorescence was subtracted at each emission wavelength.

\section{Data availability}

The data supporting the findings of this study are available within the article and its

Supplementary Information files and from the corresponding authors on request. Escherichia coli plasmids pK18 and pK19: selection of defined deletions in the 
bioRxiv preprint doi: https://doi.org/10.1101/2020.07.07.187567; this version posted July 7, 2020. The copyright holder for this preprint (which was not certified by peer review) is the author/funder. All rights reserved. No reuse allowed without permission. chromosome of Corynebacterium glutamicum. Gene 145, 69-73 (1994).

936 50. Xia, Y. et al. T5 exonuclease-dependent assembly offers a low-cost method for efficient cloning and site-directed mutagenesis. Nucleic Acids Res. 47, e15 (2018). 